\title{
A Study of the Propagation of ulf Electromagnetic Fields in Collisional, Inhomogeneous, Magnetized Plasmas
}

\author{
Joseph E. Borovsky*
}

\author{
DISCLAIMER
}

This report 'was prepared as an account of work sponsored by an agency of the United States Government. Neither the United States Government nor any agency thereof, nor any of their empluyees, makes any warranty, express or implied, or assumes any legal liability or responsibility for the accuracy, completeness, or usefulness of any information, apparatus, product, or process disclosed, or represents that its use would not infringe privately owned rights. Reference herein to any specific commercial product, process, or sirvice by trade name, trademark, manufacturer, or otherwise does not necessarily constitute or imply its endorsement, recommendation, or favnring by the United States Government or any agency thereof. The views and opinions of authors expressed berein do not necessarily state or reflect those of the United States Government or any agency thereof.

- Portions of the work described in this report were pertormed while the author was employed by The University of lowa, lowa City, IA $\mathbf{5 2 2 4 2}$. 
A STUDY OF THE PROPAGATION OF ulf ELECTROMAGNETIC

FIELDS IN COLLISIONAL, INHOMOAENEOUS, MAGNETIZED PLASMAS

by

Joseph E. Borovsky

\begin{abstract}
The propagation of ultralow-frequency (ulf) electromagnetic signals (Alfvén and magnetosonic waves) in collisional, inhomogeneous, magnetized plasmas is analyzed by numerical simulation. The problem is formulated from a Maxwe 11 -equation orbit-theory approach rather than from a magnetohydrodynamic point of vlew, and the problem is numerically treated in a fully time-dependent manner. Boundary-value-problem behavior is distinguished from initial-value-problem behavior. The propagation of twodimensional small-amplitude electromagnetic disturbances in plasmas with spatially dependent densities and in plasmas with spatially dependent conductivities is numerically simulated, and when possible, the simulations are compared with theory. Changes in the plasma density lead to change:s in the signai speed and to reflections; collisions lead to changes in the signal speed, to reflections, and to attenuations. Theoretical descriptions based upon discontinulties in the media are generally incorrect in predicting the amplitudes of signals reflected rom plasma inhomogeneities.
\end{abstract}

I. INTRODUCTION

This report documents a study of the propagation of ultralow-frequency (ulf) electromagnetic signals in the diverse media of interest to plasma 
physics, including collisionless and collisional magnetized plasmas, Inhomogeneous magnetized plasmas, and vacuums.

In plasmas, electromagnetic waves with frequencles well below the lon gyrofrequencles are referred to as mind waves, but as pointed out by $\AA_{s} t r o m, l$ these waves belong to th? same mode as vacuum electromagnetic waves. When such low-frequency wave propagates in a plasma, the wave electric fleld drives time-dependent plasma currents, and these currents produce timedependent magnetic fields that induce electric flelds that interfere with the original wave electric fleld, resulting in a wave traveling at a velocity less than the vacuum speed of light. Providing that the surrent density and charge density within a medium can be specifled in terms of the electromagnetic flelds, Maxwell's equations can be used to describe the propagation of an electromagnetic disturbance therein. Such will be the case for the plasma media of interest here. Because space plasmas can be collisional, inhomogeneous, and nonisotropic, and because propagation into planetary atmospheres will also be considered, Maxwell's equations provide a more natural description of the fleld dynamics than does the set of mhd equations.

For Inhomogeneous media and for timeodependent media, normal-mode analysis is not of use in studying the propagation of electromagnetic signals. Instead of using a Fourler-transform approach, Maxwell's equations are consolidated into one partial differential equation in two spatial varlables plus time, and that equation is numerically solved with the use of a flexible fint te-difference algorithm.2

In this report, the electromagnetic disturbances studied are of small amplitude, the plasma media not being modifled by their presence, and the properties of the media are taken to be time independent. Some of the problems that this study pertains to are the reflection, transmission, and attenuation of two-dimenslonal mhd modes propagating down on to the terrestrial lonosphere; the ability of Io-launched waves to escape the Io plasma torus; the transinission of mhd waves through the solar sorona; the coupling of magnetized accretion disks to neutron-star magnetospheres, and the Alfvén-wave heating of laboratory plasmas. This report will serve as the basis for future reports on these more spectific problens.

The formulation of the problem of electromagnetic-field propagation is developed in Section II, and the numerical code used to solve the problem is described in Section III. Section IV contalins a brief discussion about the shear and compressive polarizations of the propagating flelds, and an overview 
of the effects of the various plasma conductivities is presented in Section $V$. The simulation results for signal propagation in inhomogeneous collisional plasmas are contained in Section VI. Finally, Section VII discusses the applications of the work. Appendix A contains a derivation of the plasma conductivities; Appendix B contalns a discussion about the properties of the telegrapher's equation; Appendix C derives Alry-function-expansion reflection and transmission coefficients for mhd waves propagating into linear density ramps; Appendix $D$ develops an approximation method for describing mhd propagation in inhomogeneous plasmas; and Appendix $E$ derives impedancematching reflection and transmission coefficients for conductivity jumps in plasmas.

\section{I. ELECTROMAGNETIC-FIELN PROPAGATION IN COLLISIONAL PLASMAS}

For a diversity of media, mhd descriptions of ulf wave propagation will fail. Within magnetized plasmas, descriptions derived from the infiniteIsotropic-conductivity mhd equations ${ }^{3,4}$ will not work because the actual conductivities are finite (often near zero) and are highly anisotropic. Improvements on these mhd derivations 5 that include finite anisotropic conductivity $\vec{J}$ fall for collisionless plasmas when the displacement current is included. In the vacuum, also, the mhd equations will not suffice to describe the electromagnetic-field dynamics.

To generate a formalism for the propagation of low-frequency electromagnetic fields that is valid in a magnetosphere, lonosphere, and a tmosphere, Maxwel1's equations are employed, with the currents and charge densities speclfied in terms of the fields themselves. This formalism follows from the work of $\AA$ strom, 1 here generalized to collisional plasmas. Including the displacement current, Maxwel1's equations are

$$
\begin{aligned}
& \nabla \times \vec{B}=\frac{4 \pi}{c} \vec{J}+\frac{1}{c} \frac{\partial \vec{E}}{\partial t} \\
& \nabla \times \vec{E}=-\frac{1}{c} \frac{\partial \vec{B}}{\partial t} \\
& \nabla \cdot \vec{B}=0 \\
& \nabla \cdot \vec{E}=4 \pi \rho q,
\end{aligned}
$$


where $\vec{B}(\vec{x}, t)$ is the magnetlc induction, $\vec{E}(\vec{x}, t)$ is the electric fleld, $\vec{J}(\vec{x}, t)$ is the current density, and $\rho_{q}(\vec{x}, t)$ is the charge density. Providing that the electromagnetic flelds vary slowly compared with the lon gyrofrequency, the current density $\vec{J}$ in a cool magnetized plasma may be written as

$\vec{J}=\sigma_{p e d} \vec{E}_{\perp}+\sigma_{\|} \vec{E}_{\| l}+\sigma_{p o l} \frac{\partial E_{\perp}}{\partial t}+\sigma_{H} \hat{B}_{0} \times \vec{E}+\sigma_{c p o l} \hat{B}_{,} \times \frac{\partial E}{\partial t}$,

where $\perp$ and $\|$ refer to directions with respect to the direction of the ambient magnetic induction $\vec{B}_{0}$, and the charge density $\rho_{q}$ may be obtained from charge conservation

$$
\frac{\partial \rho_{q}}{\partial t}=-\nabla \cdot \vec{J}
$$

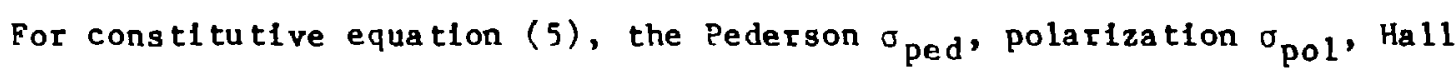
$\sigma_{H}$, cross-polarization $\sigma_{c p o l}$, and parallel $\sigma_{\|}$conductivities for a plasma are serlved using mean-free-path theory in Appendix A: they are

$$
\begin{aligned}
\sigma_{p e d} & =\frac{n_{0}^{e c}}{B_{0}}\left[\frac{v_{e} \omega_{c e}}{v_{e}^{2}+\omega_{c e}^{2}}+\frac{v_{1} \omega_{c 1}}{v_{1}^{2}+\omega_{c 1}^{2}}\right] \\
\sigma_{p o l} & =\frac{n_{0} e c}{B_{0}}\left[\frac{\omega_{c e}}{v_{e}^{2}+\omega_{c e}^{2}}+\frac{\omega_{c 1}}{v_{1}^{2}+\omega_{c 1}^{2}}\right] \\
\sigma_{H} & =\frac{n_{0} e c}{B_{0}}\left[\frac{\omega_{c e}^{2}}{v_{e}^{2}+\omega_{c e}^{2}}-\frac{\omega_{c 1}^{2}}{v_{1}^{2}+\omega_{c 1}^{2}}\right] \\
\sigma_{c p o l} & =\frac{n_{0} e c}{B_{0}}\left[-\frac{v_{e}}{v_{e}^{2}+\omega_{c e}^{2}}+\frac{v_{1}}{v_{1}^{2}+\omega_{c 1}^{2}}\right] \\
\sigma_{\|} & =n_{0} e^{2}\left[\frac{1}{m_{e} v_{e}}+\frac{1}{m_{1} \nu_{1}}\right],
\end{aligned}
$$

$n_{0}$ belng the electron number density, e the electronic charge, $B_{0}$ the magne'cic induction of the medium, $m_{e}$ and $m_{1}$ the electron and ion masses, $\omega_{c e}$ and $\omega_{c l}$ 
the electron and ion gyrofrequencies, and $v_{e}$ and $v_{1}$ the electron and lon momentum-exchange collision frequencies. The Pederson, parallel, and Hall conductivities are those commonly derived for a de plasma $0 \mathrm{hm}^{-2} \mathrm{~s} 1 \mathrm{aw}, 5,6 \overrightarrow{\mathrm{J}}=$ $\vec{\sigma}$. $\vec{E}$; the polarization and cross-polarization conductivitles give terms with $J \propto \partial E / \partial t$ rather than $J \propto E$. The effects of the various conductivities on the propagation of electromagnetic fields will be discussed at length in Section V. For an oxygen plasma within which the electron momentum-exchange collision frequency $\nu_{e}$ exceeds the lon momentum-exchange collision frequency $v_{1}$ by a factor of 100 , these conductivities are plotted as functions of $v_{1} / \omega_{c 1}$ in Figure 1 .

For a collisionless plasma, the $J_{\|}=\sigma_{\|} E_{\|}$term in relation (5) fails: the accual parallel current at one point in space depends on the time history of $E_{\|}$, on $E_{\|}$at other spatial locations, and on the details of the velocity

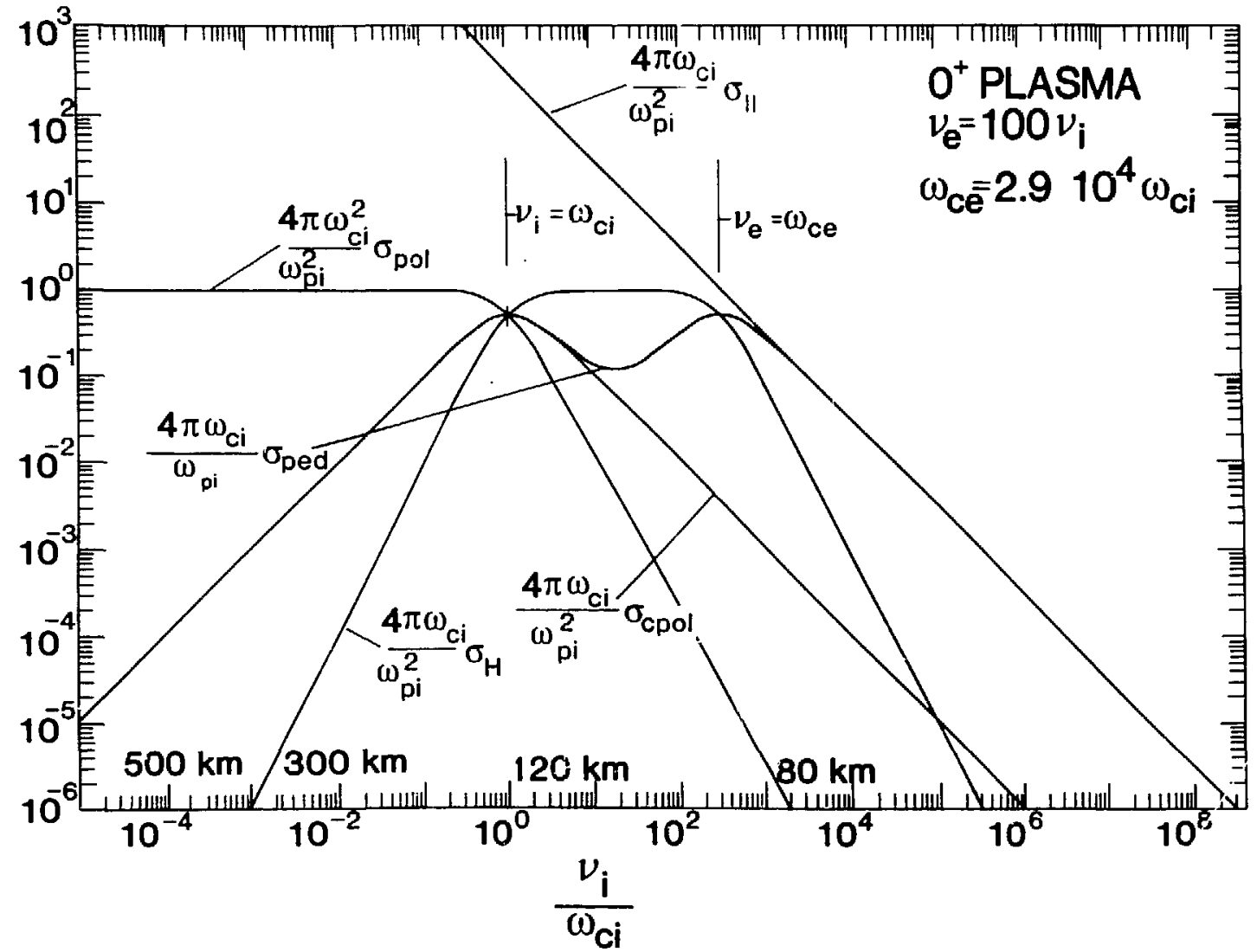

Fig. 1. For an oxygen plasma with $v_{e}=100 v_{1}$, the Pederson $\left(\sigma_{p}\right)$, parallel $\left(\sigma_{\|}\right)$, Hall $\left(\sigma_{H}\right)$, polarization $\left(\sigma_{\text {pol }}\right)$, and cross-polarization ( $\left.\sigma_{\text {cpol }}\right)$ conductivities from Equations ( $7 a$ - e) are plotted as functions of the ion collision frequericy. The numbers near the bottom of the graph pertain to the altitude (in kilometers) in the terrestrial ionosphere at which $v_{1}$ has the corresponding values. 
distribution functions. Thus, for a collisionless plasma, a realistic $\sigma_{\|}$is nonlocal in space and in time and can be only approximately obtalned. The inclusion of a nonlocal conductivity makes the electromagnetic fleld propagation problem nonlinear, since the medium is modifled by the wave. For this reason, $\sigma_{\|}$will be taken to be locai in this report. Some of the collisionless-plasma phenomena that will not be captured by this restricted treatment are particle acceleration by propagating parallel electric flelds, particle scattering by high-frequency waves driven by low-frequency fields, and the formation of electrostatic parallel electric fields.

\section{NUMERICAL SOLUTION OF THE WAVE EQUATIONS}

For each physical system in which electromagnetic-fleld propagation is simulated, the approprlate components of Maxwel1's equations (1) - (4) and the constitutive relations (5) and (6) are assembled into one scalar partial differenilal equation for $f(y, z, t)$, with $f$ being a field component or a potential. This equation is then numerically solved on a two-dimensional grid with the two grid dimensions denoted $y$ and $z$ (see Figure 2). For the flelds $\vec{E}$ and $\vec{B}$ and for sources $\vec{J}$ and $\rho_{q}$, the numerical code requires that $\partial / \partial x=0$ be taken. Further, the properties of the medium [the conductivities ( $7 a)-(7 e)$ ] must be functions only of $z$. This allows for horizontal-direction Fourler transforming $e^{1 k_{y} y}$ of the flelds, slmplifying the numerical calculation.

After the partial differential equation is Fourier transformed in the $y$ direction, it must be of the form

$$
\begin{gathered}
C_{a} \frac{\partial^{4} f}{\partial z^{2} \partial t^{2}}+C_{b} \frac{\partial^{3} \frac{z}{\partial z^{2} \partial t}}{}+C_{c} \frac{\partial^{3} f}{\partial z \partial t^{2}}+C_{d} \frac{\partial^{2} f}{\partial z^{2}} \\
+C_{e} \frac{\partial^{2} f}{\partial z \partial t}+C_{f} \frac{\partial^{2} f}{\partial t^{2}}+C_{g} \frac{\partial f}{\partial z}+C_{h} \frac{\partial f}{\partial t} \\
+C_{f} f+C_{J}=0
\end{gathered}
$$

where $f\left(k_{y}, z, t\right)$ is the $y$-Fourler transform of the fleld quantity described by the reduction of Maxwel1's equations, and where the 10 coefficlents of the equations $C_{a}, C_{b} \ldots, C_{J}$ are functions of $z, k_{y}$, and $t$. Each $k_{y}$ component of equation (8) is of the form of a fourth-order partial differential equation in $z$ and $t$. 


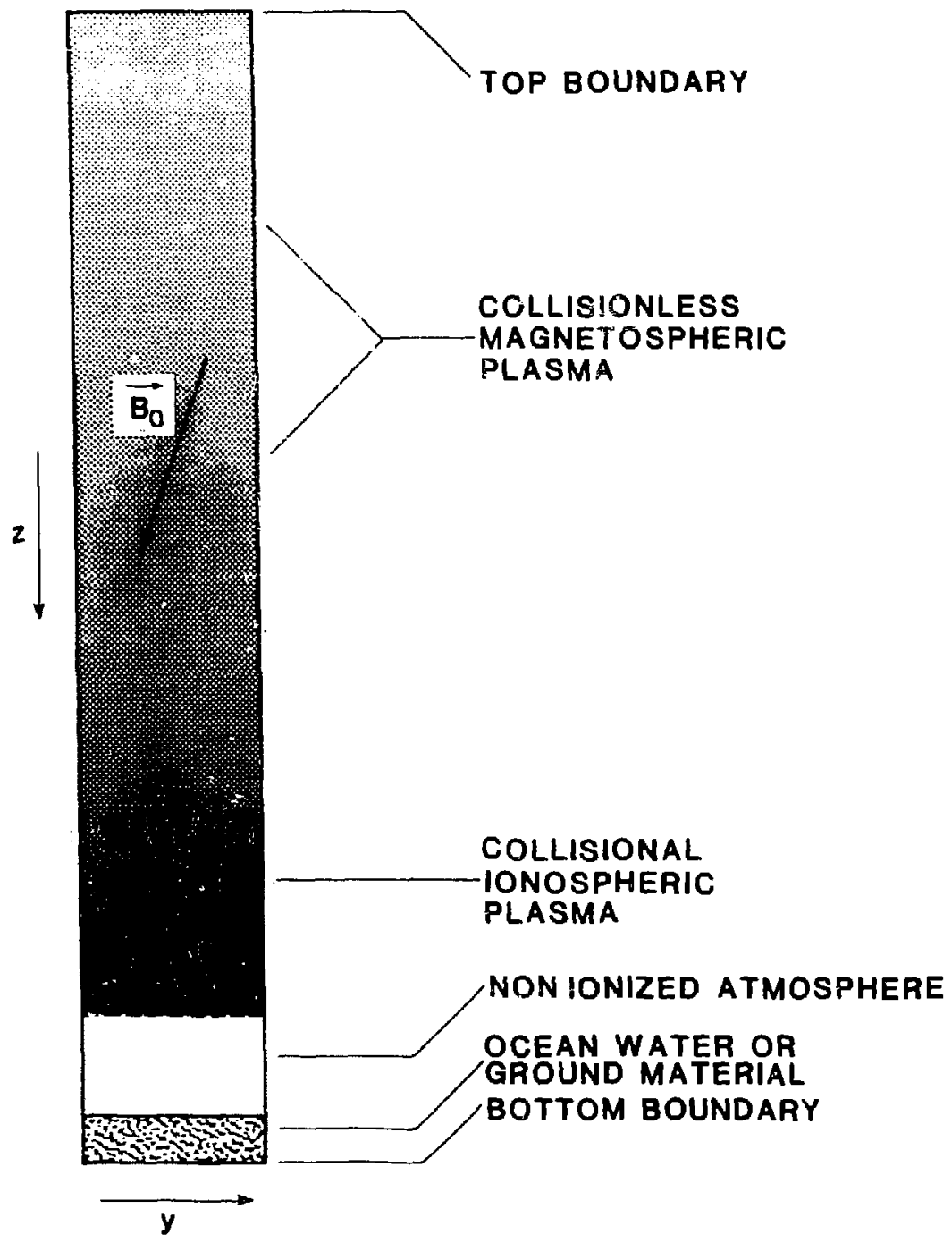

Fig. 2. The geometry of the two-dimensional simulation system (with the medium labeled according to the terrestrlal magnetosphere-1onospherea tmosphere-ground system).

The numerical code uged to follow the time evolution of the system is described in Borovsky.2 The numerical algorithm of the code is versatile and can be set for high accuracy or for computational speed. Rather than solving the problem only in the titie domain or only in the space domain, equation (8) is finite differenced in both $z$ and $t$ and then is solved implicitly for the new-time values of $f$ on the $z-k_{y} g r i d$. The code has been successfully tested for both hyperbolic (wave) and parabolic (diffusion) equations. This testing is necessary since the nature of electromagnetic signals in collisional plasmas is sometimes (or somewhere) hyperbolic and sometimes (somewhere) parabolic. 
Because these modes in general will obey different wave equations, it is convenient to describe the propagation of the component of the electric field normal to the y-z plane (see Figure 2) separately from the propagation of the electric-fleld component that is coplanar with $y-z$. If the external magnetic induction $\vec{B}_{0}$ lies in the $y-z$ plane, then the portion of the disturbance with electric field $\vec{E}$ normal to the simulation plane $\left[\vec{E}=\left(E_{x}, 0,0\right)\right]$ is denoted as a compressional disturbance, and the portion with electric field $\vec{E}$ in the plane $\left[\vec{E}=\left(0, E_{y}, E_{z}\right)\right]$ is denoted as a shear disturbance. The shear and compressional nomenclatures pertain to the bulk motion of any collisionless plasma disturbed by these modes. If $\vec{E}$ lies in the plane, then particle $\vec{E} \times \overrightarrow{B_{0}}$ drifts are normal to the plane, and since the operator $\nabla=\left(0, \partial_{y}, \partial_{z}\right)$ lies in the $y-z$ plane, $\vec{v} \cdot 7=0$; hence, the disturbance is shearlike. Similarly, if E is normal to the $y-\pi$ plane, then particle $\vec{E} \times \vec{B}_{0}$ drifts are In the plane and $\vec{v}, \quad \nabla \neq 0$; hence, the disturbance is compressional. In the absence of Hall conductivities (which couple the shear and compressive modes), the disturbance magnetic Induction $B$ is normal to both $\vec{E}$ and $\nabla$; therefore, with $B_{0}$, in the $y-z$ plane, for the compressive mode $\vec{B}, \vec{B}_{0} \neq 0$ and for the shear mode $\vec{B}_{B_{0}} \vec{B}_{0}=0$. In a cold, collisionless, magnetized piasma, the shear (Alfven) mode and the compressive (fast magnetosonic) mode are the only existing electromagnetic modes with frequency $w$ much less than the Ion gyrofrequency $\omega_{c i}$. In Section V.A it will be shown that their propagation is determined by two different equations--the compressive mode propagates isotropically and the shear mode is guided along the ambient magretic field.

\section{EFFECTS OF THE CONDUCTIVITIES ON ulf FIELD PROPAGATION}

Each term in the constitutive equation (5) contributes differently as a source in Haxwel1's equations. The origin of these terms and their effects on the propagation of low-frequency electromagnetic fields in homogeneous collisional plasmas are Investigated in this section. The effects of spatially varying conductivities are deferred to section VI. 
The polarization conductivity ( $7 b$ ) describes the effects of collisions on the polarization drifts of electrons and lons. In the absence of collisions, these guiding-center-drift velocities are given by $\vec{v}=\left(\mathrm{mc}^{2} / \mathrm{eB}_{0}^{2}\right)\left(\mathrm{dE}_{\perp} / \mathrm{dt}\right)$. Because of their larger mass, the lons contribute much more to the polarization current than the electrons do. Collisions act to impede the polarization drifts of both lons and electrons; hence, they reduce the polarization current. This is reflected by a decrease in the polarization conductivity $\sigma_{\text {pol }}$ as the collision frequency increases (see Figure 1 ).

The role of the polarization conductivity $\sigma_{\text {pol }}$ on electromagnetic-field propagation is investigated by taking the collisionless limlts $\sigma_{\|} \rightarrow \infty$, $\sigma_{\text {ped }}+$ $0, \sigma_{H} \rightarrow 0$, and $\sigma_{\text {cpol }} \rightarrow 0$; equation $(5)$ then becomes $J^{\prime}=\sigma_{\left[\gamma_{0}\right.} \partial \vec{E}_{i} / \partial t$. Because $J_{\|}$is infinite, $\underset{+}{+}$ is taken to be zero. Ampere-s law ( 1 ) and Faraday's law (2) combine with $\vec{J}=\sigma_{\text {pol }} \overrightarrow{\partial E_{\perp}} / \partial t$ to yield

$$
\nabla\left(\nabla_{\perp} \cdot \overrightarrow{\mathrm{E}}_{\perp}\right)-\nabla^{2} \overrightarrow{\mathrm{E}}_{\perp}=-\frac{1+4 \pi \sigma_{\mathrm{pol}}}{\mathrm{c}^{2}} \frac{\partial^{2} \overrightarrow{\mathrm{E}}_{\perp}}{\partial \mathrm{t}^{2}} .
$$

The perpendicular electric fleld can be separated into two components, one with $\overrightarrow{\mathrm{E}}_{\perp} \perp \nabla_{\perp}$ (compressive) and one with $\overrightarrow{\mathrm{E}}_{\perp} \| \nabla_{\perp}$ (shear):

$$
\frac{\partial^{2} \vec{E}_{\perp}}{\partial t^{2}}-\frac{c^{2}}{I+4 \pi \sigma} \nabla_{\text {pol }}^{2} \vec{E}_{\perp}=0
$$

and

$$
\frac{\partial^{2} \vec{E}_{1}}{\partial t^{2}}-\frac{c^{2}}{1+4 \pi \sigma p o l} \nabla_{\|}^{2} E_{i}=0 \text {. }
$$

Both (10) and (11) are hyperbolic equations with characteristic velocities

$$
v_{c}= \pm \frac{c}{\left(1+4 \pi \sigma_{p o l}\right)^{I / 2}} .
$$


Equation (10) describes the 1sotropic propagation with velocity $v_{c}$ of the $\stackrel{+}{E}_{\perp}$ $\nabla_{\perp}$ component; this $1 s$ the cold magnetosonic (fast) mode (see Ref. 1 and Section 7.12 of Ref. 7). Equation (11) describes the guided propagation with velocity $v_{c}$ along $\vec{B}_{0}$ of the $\vec{E}_{\perp} \perp \nabla_{\perp}$ component; this is the shear Alfven mode (sec Ref. 1 and Section 7.11 of Ref. 7).

The effects of collisions on the characteristic velocity for lowfrequency electromagnetic-field propagation in plasmas can be determined as follows. The polarization conductivity can be written as $\sigma_{\text {pol }}=\left(c^{2} / 4 \pi v_{A}^{2}\right) \xi$, where, from equation $(7 b), \xi=\left(\omega_{c e} \omega_{c t}\right) /\left(\nu_{e}^{2}+\omega_{c e}^{2}\right)+\left(\omega_{c 1}^{2}\right) /\left(\nu_{1}^{2}+\omega_{c i}^{2}\right)$, and where $v_{A}=B_{0} /\left(4 \pi n_{0}\left(m_{1}+m_{e}\right)\right)^{1 / 2}$ is the Alfven velocity. The quantity $\xi$ decreases monotonicaliy $\overline{\text { rixuin }} 1$ íj $\bar{U}$ as the moinentum-transfer collision frequencles $\nu_{e}$ and $\nu_{i}$ in the plasma go from 0 (collisionless) to o (very collisional). With $\sigma_{\text {pol }}$ given by $\left(c^{2} / 4 \pi v_{A}^{2}\right) \xi$, the characteristic velocity (12) becomes

$$
v_{c}= \pm \frac{v_{A} c}{\left(v_{A}+c^{2} \xi\right)^{1 T 2}}
$$

In a collision less plasma, $\xi+1$ and relation (13) becomes

$$
v_{c}= \pm \frac{v_{A} c}{\left(v_{A}^{2}+c^{2}\right)^{I T L}},
$$

the speed of propagation of a low-frequency electromagnetic signa 1 in a cold, collisionles,, magnetized plasma. If $v_{A} \ll c$, then $v_{c} \cong v_{A}$ and Alfven-speed propagation is obtained. If $v_{A}+\infty$ (because $n_{0}+0$ ), then $v_{c}+c$, and the signal propagates at the vacuum speed of light. Thus, the vacuum electromagnetic wave and low-frequency mhd waves are the same modes (and there is no need to consider mode conversion at the lonosphere/atmosphere interface).

In a highly collistonal plasma, $\xi+0$ and relation (13) becomes

$$
v_{c}+ \pm c,
$$

the speed of propagation of a low-frequency electromagnetic signal in a collisional plasma approaching the vacuum velocity of light. By comparing 
relation (15) with relation (14), the reader can see that collisions act to increase the chazacteristic velocity of the plasma. This increase is reasoned as follows. The effect that adding the displacement current $(1 / \mathrm{c})(\partial E / \partial t)$ to Ampere-s law had was to lower the electromagnetic-propagation speed down from - (instantaneous) to c (see Section VI of Ref. 8). The polarization current, as well as the displacement current, has the form $\vec{J} \propto \partial \vec{E} / \partial t$. Thus, in Ampere's Law the polarization current acts like the displacement current: the polarization current reduces the propagation speed. In a collisionless plasma with $v_{A} \ll c$, the effect of the polarization curzent in Ampere's law is to slow a signal down from c. to $v_{A}$. If collisions are added to the plasma, then the polarization current is impeded, and the speed of propagation increases from $v_{A}$ back toward $c$.

B. The Pederson and Parallel Conductivities: Damping and Guiding

The Pederson conductivity (7a) describes the plasma current driven across a unfiorm magnetic induction by a time-scationary electric field. If collisions are present, then the gyro-orbits of plasma particles are disrupted and the particies will have net drifts parallel or antiparallel to $\overrightarrow{\mathrm{E}}_{\perp}$. These drift velocities are maximized when the momentum-exchange collision frequencles are equal to the gyrofrequencles, a balance occurring between gyro-orbit disruption and collisional mobility. Differences between the lon and electron collisional drift velccities result in currents in the direction of $\overrightarrow{\mathrm{E}}_{\perp}$, the current density being related to $\overrightarrow{\mathrm{E}}_{\perp}$ through the Pederson conductivity $\sigma_{\text {ped }}$. As can be seen in Figure $1, \sigma_{\text {Ped }}$ has maxima when $\nu_{e}=\omega_{c e}$ and when $v_{1}=\omega_{c i}$.

The parallel conductivity (7e) describes the effects of collisions on the motion of charged particles parallel to $\vec{B}_{0}$ in the presence of a timestationary electric field. In a collisional plasma, the electrons and ions reach terminal parallel velocities that depend on the strength of the applied parallel electric field $E_{\|}$and on the colision frequencles. In such a plasma, the parallel current density is related to the parallel electric field through the parallel conductivity $\sigma_{\mathbb{1}}$ (see Figure 1).

Finite Pederson and parallel conductivities lead to the ohmic damping of electromagnetic disturbances in plasmas, and the value of the parallel conductivity determines whether or not the propagation of a shear electromagnetic disturbance is guided along a magnetic field. Tíe effects that these conductivities produce on low-frequency electromagnetic 
disturbances can be 1 solated by taking $\sigma_{\mathrm{H}_{+}}+0$ and $\sigma_{+}$cpol +0 , which leaves the constitutive relation (5) In the form $\vec{J}=\sigma_{\text {ped }} \vec{E}_{\perp}+\sigma_{\|} \vec{E}_{\|}+\sigma_{\text {pol }} \partial \vec{E}_{\perp} / \partial t$; inserting this relation into the Faraday-Ampere combination (1) and (2) ylelds a vector equation that can be reduced to two coupled equations, one perpendicular to $\overrightarrow{\mathrm{B}}_{0}$ and one parallel to $\overrightarrow{\mathrm{B}}_{0}$,

$$
\nabla_{\perp}\left(\nabla_{\perp} \cdot \vec{E}_{\perp}+\nabla_{\|} E_{\|}\right)-\nabla^{2} \vec{E}_{\perp}=-\frac{4 \pi \sigma_{\text {ped }}}{c^{2}} \frac{\partial \vec{E}_{\perp}}{\partial t}-\frac{1+4 \pi \sigma_{\text {pol }}}{c^{2}} \frac{\partial^{2} \vec{E}_{\perp}}{\partial t^{2}}
$$

and

$$
\nabla_{\|}\left(\nabla_{\perp} \cdot \overrightarrow{\mathrm{E}}_{\perp}+\nabla_{\|} \mathrm{E}_{\|}\right)-\nabla^{2} \overrightarrow{\mathrm{E}}_{\|}=-\frac{4 \pi \sigma_{\|}}{\mathrm{c}^{2}} \frac{\partial \overrightarrow{\mathrm{E}}_{\|}}{\partial \mathrm{t}}-\frac{1}{\mathrm{c}^{2}} \frac{\partial^{2} \overrightarrow{\mathrm{E}}_{\|}}{\partial \mathrm{t}^{2}} .
$$

The perpendicular and parallel components of the electric fleld are coupled through $\nabla_{\perp} \nabla_{\|}$terms. For simplification, the electric field of the disturbance is separated into a compressional component $\vec{E}_{\perp} \perp \nabla_{\perp}$ and a shear component $\vec{E}_{\perp} \|$ $\nabla_{\perp}$, and eacin component is analyzed separately for the effects of the parailel and Pederson conductivities.

1. Effects on Compressive Disturbances. For $\quad \overrightarrow{\mathrm{E}}_{\perp} \perp \nabla_{\perp} \quad$ (compressive: disturbance), equation (16) contains a component parallel to $\vec{E}_{\perp}$ and a component parallel to $\nabla_{\perp}$. The $\nabla_{\perp}$-directed component is $\nabla_{\perp} \nabla_{\|} E_{\|}=0$, giving $E_{\|}$ $=0$. With $E_{\|}=0$, equation (17) and the $\vec{E}_{\perp}$-directed component of equation (16) become the decoupled set

$$
\frac{\partial^{2} \vec{E}_{1}}{\partial t^{2}}-\frac{c^{2}}{1+4 \pi \sigma_{p o 1}} \nabla^{2} \vec{E}_{\perp}=-\frac{4 \pi \sigma_{p e d}}{1+4 \pi \sigma_{p o 1}} \frac{\partial \vec{E}_{1}}{\partial t}
$$

and

$$
\frac{\partial^{2} \overrightarrow{E_{\|}}}{\partial t^{2}}-c^{2} \nabla_{\perp}^{2} \vec{E}_{\|}=-4 \pi \sigma_{\|} \frac{\partial \vec{E}_{\|}}{\partial t} \text {. }
$$

Both equations have the telegrapher's equation form (see Appendix $B$ ). Envation (18a) describes the isotroplc propagation at velocity $c /\left(1+4 \pi \sigma_{p o l}\right)^{1 / 2}$ of the compressive component of $\vec{E}_{1}$. For this component, the Pederson-conductivity damping acts the same as an isotropic resistivity in a 
nonmagnetized medium (see Section 798 of Ref. 9 and Section 7.7 of Ref. 10). According to the onalysis of Appendix B, only initial-value discurbances with scale sizes $\lambda<\left(c / \sigma_{\text {ped }}\right) \sqrt{1+4 \pi \sigma \text { pol }}$ propagate, and when there is propagation, disturbances are damped in time. Numerical simulations of this damping appear In Figure 3, where the amplitude of a truncated sine-wave pulse propagating in a homogeneous colifsional plasma is plotted as a function of time for various values of $\sigma$ ped. In each computer run, the pulse was initiated $w i$ th $\partial / \partial y=0$; hence, $\nabla^{2} \rightarrow \partial^{2} / \partial z^{2}$ in equation (18a).

Equation (18b) describes the propagation of $\overrightarrow{\mathrm{E}}_{\|}$at the speed of $11 \mathrm{ght} \mathrm{c}$ across $\vec{B}_{0}$. This is the low-frequency ordinary mode, ohmically damped by collisions acting on the parallel motion of charged particles. (In a truly collisionless plasma, the ordinary electromagnetic mode will not propagate unless $\omega>\omega_{\text {pe' }}$ where $\omega_{\text {pe }}$ is the electron-plasma frequency.) Analyzing equation (18b) with Appendix B treatment, it is found that an initial-value ordinary-mode disturbance with scale size $\lambda$ will propagate provided that $\lambda<$ $c / \sigma_{\|}$. By comparing equations (18b) and (B7), and by using relation (7e), the

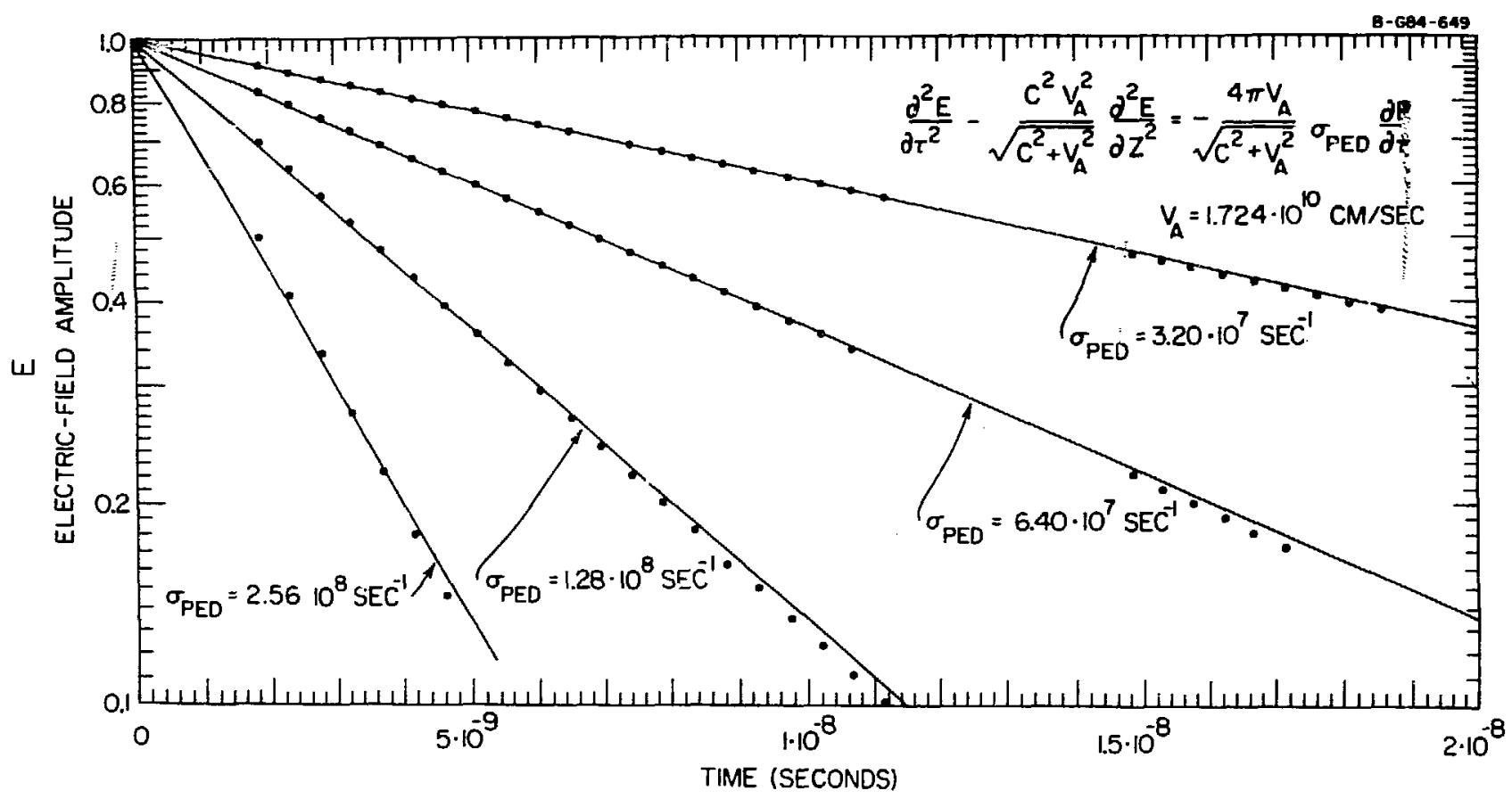

Fig. 3. The damping of compressive-polarization truncated-sine-wave pulses as they prcpagate in homogeneous plasmas with differing values of the Pederson conductivity. Any simulations of telegraphic-equation propagation appear as they do in this figure. The points are from simulations and the 1 ines are from equation ( $18 \mathrm{a})$. 
collisional-plasma skin deptì for the ordinary-mode boundary-value problem may be written in the $\sigma_{\|} \gg \omega$ case as

$$
\delta=\sqrt{2} \frac{c}{\omega_{p e}}\left(\frac{\nu_{e}}{\omega}\right)^{1 / 2} .
$$

This differs by a factor of $\sqrt{2 \nu / \omega}$ over the collisionless plasma skin depth $\delta=c / \omega_{\text {pe }}($ see Section 7.5 of $\operatorname{Ref} .10)$.

2. Effects on Shear Disturbances. For $\vec{E}_{1} \| \nabla_{1}$ (shear disturbance), equation (16) has only one component; this component and equation (17) become the coupled set

$$
\begin{aligned}
& \frac{\partial^{2} E_{\perp}}{\partial t^{2}}-\frac{c^{2}}{1+4 \pi \sigma p o l} \nabla_{\|}^{2} E_{\perp}+\frac{4 \pi \sigma p e d}{1+4 \pi \sigma p o l} \frac{\partial E_{1}}{\partial t}=-\frac{c^{2}}{1+4 \pi \sigma p o l} \nabla_{\perp} \nabla_{\|} E_{\|} \\
& \frac{\partial^{2} E_{\|}}{\partial t^{2}}-c^{2} \nabla_{\perp}^{2} E_{\|}+4 \pi \sigma_{\|} \frac{\partial E_{\|}}{\partial t}=-c^{2} \nabla_{\perp} \nabla_{\|} E_{\perp} .
\end{aligned}
$$

Equation (20a) describes the guided propagation of the shear component of $\Sigma_{\perp}$ (Alfvern mode), damped by nonzero $\sigma_{\text {ped }}$ and coupled to $E_{\|}$, and equation (20b) describes the propagation of $E_{\|}$perpendicular to $\vec{B}_{0}$ (ordinary mode), damped by noninfinite $\sigma_{\|}$, and coupled to $E_{\perp}$. Equations (20a) and (20b) are combined Into a single equation by operating on equation ( $20 \mathrm{~b}$ ) with $\nabla_{1} \nabla_{\|}$and then using equation $(20 a)$ to eliminate $\nabla_{1} \nabla_{\|} E_{\|}$: the result is

$$
\begin{aligned}
\frac{1}{4 \pi \sigma_{\|}} \frac{\partial^{3} E_{1}}{\partial t^{3}} & +\left(1+\frac{\sigma_{\text {ped }}}{\sigma_{\|}} \frac{1}{1+4 \pi \sigma_{\text {pol }}}\right) \frac{\partial^{2} \mathrm{E}_{\perp}}{\partial \mathrm{t}^{2}} \\
& -\left(\frac{c^{2}}{4 \pi \sigma_{\|}} \nabla_{\perp}^{2}+\frac{1}{1+4 \pi \sigma_{\text {pol }}} \frac{c^{2}}{4 \pi \sigma_{\|}} \nabla_{\|}^{2}-\frac{4 \pi \sigma_{\text {ped }}}{1+4 \pi \sigma_{\text {pol }}}\right) \frac{\partial \mathrm{E}_{\perp}}{\partial \mathrm{t}} \\
& -\left(\frac{\sigma_{\text {ped }}}{\sigma_{\|}} \frac{\mathrm{c}^{2}}{1+4 \pi \sigma_{\text {pol }}} \nabla_{\perp}^{2}+\frac{c^{2}}{1+4 \pi \sigma_{\text {pol }}} \nabla_{\|}^{2}\right) \mathrm{E}_{\perp}=0,
\end{aligned}
$$


which describes the coupled shear-ordinary mode in a collisional plasma.

If $\sigma_{\|}+\infty$, then the effects of $\sigma_{\text {ped }}$ on the shear mode are clearly seen. When this limit is used, which is equivalent to taking the $E_{\|}+01$ imit, equation ( 21 ) becomes

$$
\frac{\partial E_{\perp}}{\partial t^{2}}-\frac{c^{2}}{1+4 \pi \sigma_{p o l}} \nabla_{\|}^{2} E_{\perp}=-\frac{4 i \sigma \sigma_{p e d}}{1+4 \pi \sigma_{p o l}} \frac{\partial E_{\perp}}{\partial t},
$$

again the telegrapher's equation (see Appendix B). Equation (22) describes the ohmic damping with time of the shear $E_{\perp}$ disturbance guided along $\vec{B}_{0}$.

The magnitude of the parallel conductivity $\sigma_{\|}$determines whether a shearmode disturbance $w 111$ be guided along $\vec{B}_{0}$ or whether it will propagate isotropically. If the plasma is collisionless, $\sigma_{\text {ped }}+0, \sigma_{\|} \rightarrow \infty$, and $\sigma_{\text {pol }}+$ $c^{2} / 4 \pi v_{A}^{2}$, and the $\partial^{3} E_{1} / \partial t^{2}$ anc $\partial E_{1} / \partial t$ terms drop out of equation (21) leaving

$$
\frac{\partial^{2} E_{\perp}}{\partial t^{2}}-\frac{c^{2} v_{A}^{2}}{c^{2}+v_{A}^{2}} \nabla_{\|}^{2} E_{\perp}=0 ;
$$

this equation describes the guided propagation of a disturbance along $\vec{B}_{0}$ at the modified Alfven velocity $\pm c v_{A} i \sqrt{c^{2}+v_{a}^{2}}$. Conversely, in the complete absence of plasma, $\sigma_{\text {ped }}+0, \sigma_{\|}+0$, and $\sigma_{\text {pol }}+0$, and the $\partial^{2} E_{\perp} / \partial t^{2}$ and $E_{\perp}$ terms drop out of equation (21) leaving (after one-time integration)

$$
\frac{\partial^{2} E_{\perp}}{\partial t^{2}}-c^{2} \nabla^{2} E_{\perp}=0 ;
$$

this equation describes the isotropic propagation of a disturbance at the vacuum speed of light. The transition between guided and isotropic propagation is quantified by considering $\nabla_{\|}=0$ in equation (21); in this case, the abserce of propagation perpendicular to $\vec{B}_{0}$ indicates guiding along $\vec{B}_{0}$, and the presence of propagation perpendicular to $\vec{B}_{0}$ indicates a lack of guiding. For $\nabla_{\|}=0$, equation (21) becomes 


$$
\begin{aligned}
\frac{1}{4 \pi \sigma_{\|}} \frac{\partial^{3} E_{1}}{\partial t^{3}}+\left(1+\frac{\sigma_{\text {ped }}}{\sigma_{\|}} \frac{1}{i+4 \pi \sigma_{\text {pol }}}\right) \frac{\partial^{2} E_{1}}{\partial t^{2}}-\left(\frac{c^{2}}{4 \pi \sigma_{\|}} \nabla_{\perp}^{2}-\frac{4 \pi \sigma_{\text {ped }}}{1+4 \pi \sigma_{\text {pol }}}\right) \frac{\partial E_{1}}{\partial t} \\
-\frac{\sigma_{\text {ped }}}{\sigma_{\|}} \frac{c^{2}}{1+4 \pi \sigma_{\text {pol }}} \nabla_{\perp}^{2} E_{\perp}=0 .
\end{aligned}
$$

When $\sigma_{\sharp}$ and $\sigma_{\text {ped }}$ do pot vary perpendicular to $\vec{B}_{0}$, equation (25) can be normalmode analyzed $e^{1\left(k_{1} \cdot x_{1} w t\right)}$ to yleld the dispersion relation

$$
\left(\omega+1 \frac{4 \pi \sigma_{\text {ped }}}{1+4 \pi \sigma_{\text {pol }}}\right)\left(\omega^{2}+14 \pi \sigma_{\|} \omega-c^{2} k_{1}^{2}\right)=c \text {. }
$$

For $k_{\perp}$ real (initial-value problem), relation (26) has three solutions:

$$
\begin{aligned}
& \omega=-1 \frac{i \pi \sigma_{\text {ped }}}{1+4 \pi \sigma_{p \circ 1}}, \\
& \omega=+k_{1} c\left(1-\frac{1}{4} \frac{\left(1 \pi \sigma_{\|}\right)^{2}}{k_{1}^{2} c^{2}}\right)^{1 / 2}-12 \pi \sigma_{1},
\end{aligned}
$$

and

$$
\omega=-k_{1} c\left(1-\frac{1}{4} \frac{\left(4 \pi \sigma_{\|}\right)^{2}}{k_{1}^{2} c^{2}}\right)^{1 / 2}-12 \pi \sigma_{\|} \text {. }
$$

Solution (27a) ylelds the exponential Pederson-conductivity damping with time of a $k_{1}=0$ (spatially uniform) shear-E field. Solutions (27b; and (27c) propagate perpendicular to $\vec{B}_{0}$ when

$$
\sigma_{\|}<\frac{\left|k_{1}\right| \mathbf{c}}{2 \pi} .
$$


When relation (28) is satisfied, field-aligned guiding of a shear-mode disturbance is lost. In that case, the phase velocity perpendicular to $\vec{B}_{0}$ is $\mathbf{v}_{\mathrm{ph}}= \pm c\left[1-\left(4 \pi \sigma_{\|} / 2 \mathrm{k}_{\perp} \mathrm{c}\right)^{2}\right]$, and the damping decrement is $\omega_{1 \mathrm{mag}}=-2 \pi \sigma_{H}$. For a boundary-value problem ( $\omega$ rea 1 ), equation (26) has two solutions for $k_{\perp}$, and a perpendicular skin depth $\delta$ for the shear mode can be obtalned:

$$
\delta=\frac{1}{\mathrm{k}_{\text {Imag }}}=\sqrt{2} \frac{\mathrm{c}}{\omega}\left[\left[1+\left(\frac{4 \pi \sigma_{\|}}{\omega}\right)^{2}\right]^{1 / 2}-1\right\}^{-1 / 2}
$$

As expected, for $\sigma_{\|}+0$, the skin depth $\delta+\infty$, and the no-plasma case of isotropic propagation without damping is obtained. For $\sigma_{\|} \gg \omega$, relation (29) reduces to relation (19). In the boundary-value problem, there is always some propagation of the signal across $\vec{B}_{0}$.

The damping of shear-mode disturbances by $\sigma_{\|}$is isolated by taking $\sigma_{\text {ped }}=$ 0 in equation (21). For $\sigma_{\|} \gg \partial / \partial t$, the remaining $\partial^{3} E_{\perp} / \partial t^{3}$ and $\partial E_{\perp} / \partial t$ terms can be 1gnored. By a normal-mode analysis that assumes $w=\omega_{0}+\varepsilon$, where $\omega_{0}$ solves the $\sigma_{\|}+\infty 11 \mathrm{mit}$ of equation $(21)$ and where $|\varepsilon| \ll\left|\omega_{0}\right|$, it can be shown that, to order $\omega_{o} / \sigma_{\|}$, the $\partial^{3} E_{\perp} / \partial t^{3}$ and $\partial E_{\perp} / \partial t$ terms not only are small but also cancel. each other. The result is

$$
\frac{\partial^{2} E_{\perp}}{\partial t^{2}}-\frac{c^{2}}{1+4 \pi \sigma p o l} \nabla_{\|}^{2} E_{\perp}=\frac{c^{2}}{4 \pi \sigma_{\|}} \nabla_{\perp}^{2} \frac{\partial E_{\perp}}{\partial t},
$$

which describes the guided propagation of $E_{\perp}$ a long $\vec{B}_{0}$, the $E_{\|}-E_{\perp}$ coupling implicit in the damping term. The parallel conductivity $\sigma_{\|}$acts to damp out portions of the disturbance that have strong perpendicular gradients. Thus, a disturbance spreads out in the direction perpendicular to $\vec{B}_{0}^{+}$as 1 t propagates along $\vec{B}_{0}$; as time $t \rightarrow \infty$, only a plane wave remains. Figure 4 contains a simulation of a two-dimensional Alfven-wave pulse that is guided along the ambient magretic induction $\vec{B}_{0}=B_{0} \hat{z}$ in a uniform plasma with a finite parallel conductivity. At early times (top) the pulse is clearly two dimensional, but with time the pulse spreado perpendicular to $\vec{B}_{0}$, evolving into a plane wave (bottom). 


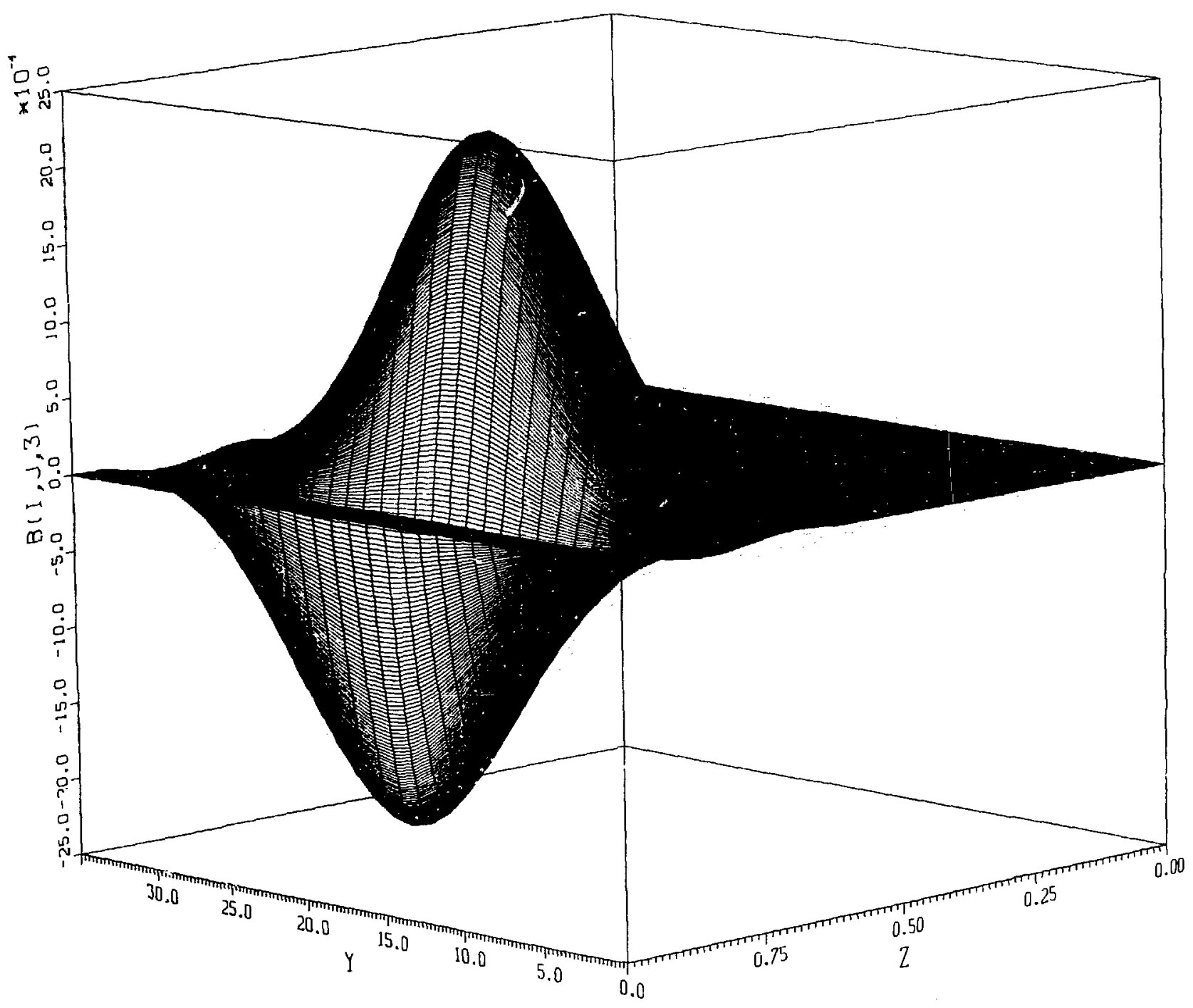

Fig. 4. A guided two-dimensional shear-mode pulse propagating in a medium with finite parallel conductivity. The ambient magnetic induction is in the $z$-direction. The numerical system is $1200 \times 32$ grid points, with equal grid spacing in the $z$ - and $y$-directions. 


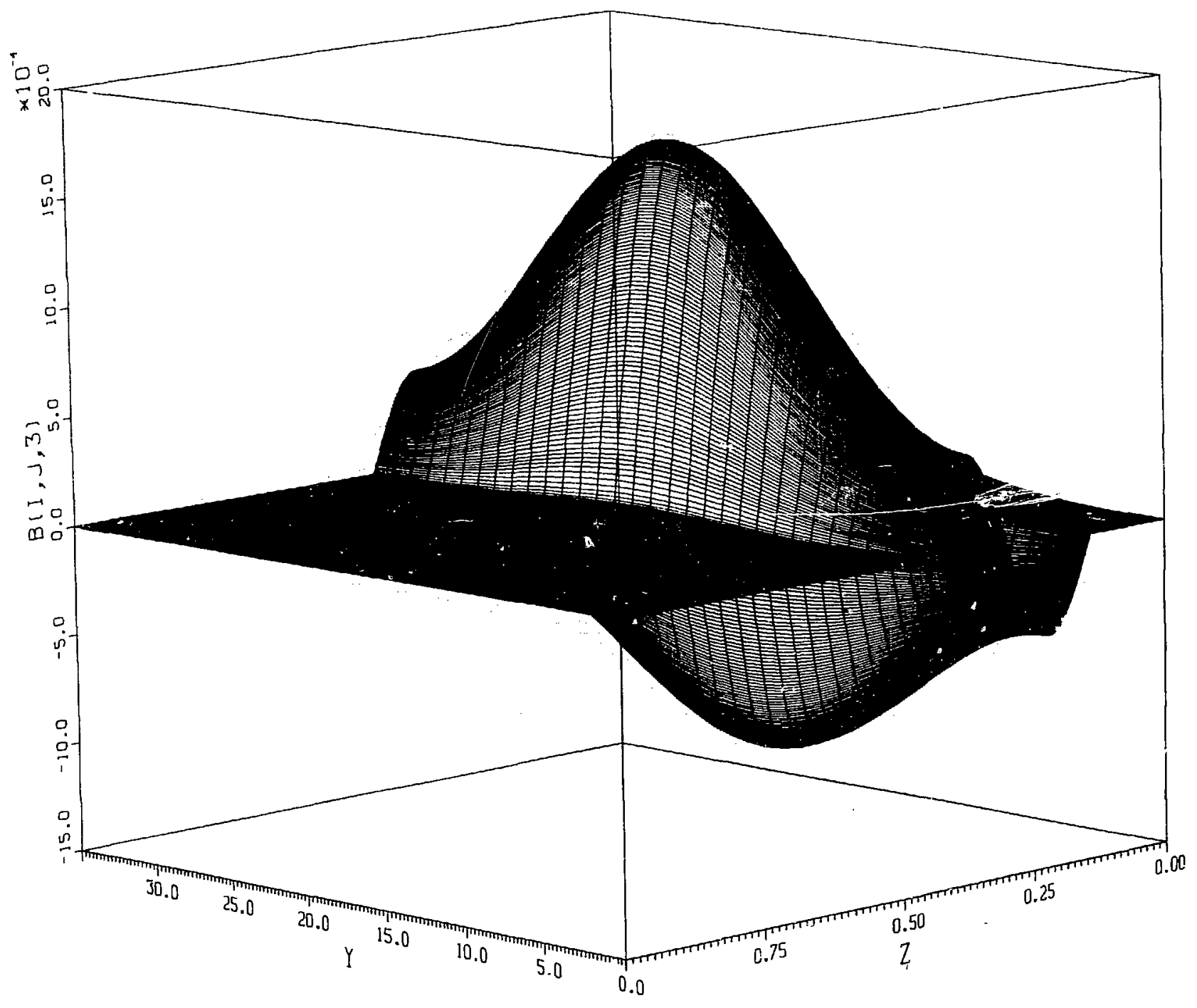

Fig. 4 (continued). 


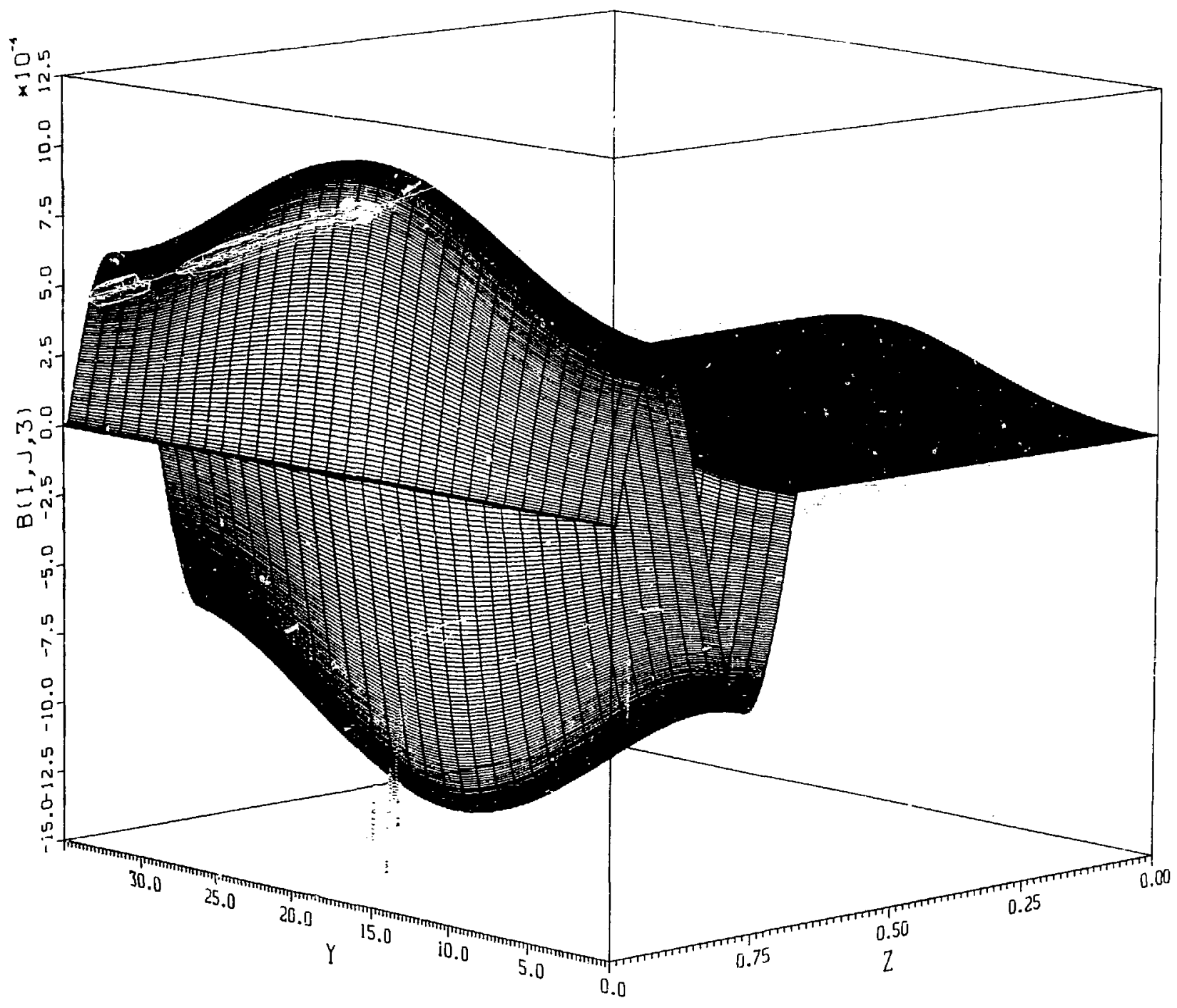

Fig. 4 (continued). 
The Hall conductivity (7c) arises from the collisional disruption of ion and electron $\vec{E} \times \vec{B}$ drifting in magnetized plasmas. In general, collisions distupt the ion drifting more than the electron drifting, and the resulting current is in the $\stackrel{+}{-} \times \stackrel{+}{B}$ direction.

For a linearly polarized electromagnetic wave propagating parallel or antiparallel to a magnetic induction $\vec{B}_{O}$, the addition of a Hall current density $\vec{J}_{H}=\sigma_{H_{0}} \hat{B} \times \vec{E}$ results in an elliptically polarized propagating current density. If the Hal1 conductivity $\sigma_{H}>0$, then for waves propagating in the direction of $\vec{B}_{O}$, the elliptically polarized current-density rector has positive helicity; and for waves propagating antiparallel to $\vec{B}_{0}$, the current vector has negative helicity. Accordingly, the plane of polarization of the electromagnetic wave rotates in the direction of electron gyromotion about $\overrightarrow{\mathrm{B}}_{0}$. The effects are quantified by keeping only the Hall and polarization currents in constitutive relation (5), the Ampere-Faraday combination (1) and yle1ding

$$
\nabla \times \nabla \times \overrightarrow{\mathrm{E}}=-\frac{4 \pi \sigma \text { pol }}{\mathrm{c}^{2}} \frac{\partial^{2} \overrightarrow{\mathrm{E}}_{1}}{\partial \mathrm{E}^{2}}-\frac{1}{\mathrm{c}^{2}} \frac{2^{2} \overrightarrow{\mathrm{E}}}{\partial \mathrm{t}^{2}}-\frac{4 \pi \sigma_{\mathrm{H}}}{\mathrm{c}^{2}} \hat{\mathrm{B}}_{0} \times \overrightarrow{\mathrm{E}}_{\perp} .
$$

To simplify the analysis, only propagation parallel to the magnetic induction $\vec{B}_{0}$ is considered. In this case, the parallel and perpendicular electric-field components decouple; since the parallel component is not affected by $\sigma_{H}$, only the perpendicular component is considered. Taking ${ }_{B} f(k z-\omega t)$ and assuming an infinite, homogeneous plasma, normal-mode analysis $e^{1(k z-\omega t)}$ of equation (31) ylelds the coupled set of equations for the perpendicular components of the electric field $\left(\omega^{2}-k^{2} v_{c}^{2}\right) E_{x}=-1 \omega K_{H} E_{y}$ and $\left(\omega^{2}-k^{2} v_{c}^{2}\right) E_{y}=+1 \omega k_{H} E_{x}$, where $v_{c}=c / \sqrt{1+4 \pi \sigma}$ pol is the characteristic velocity of equation (31) and where $\kappa_{H}$ is defined as $\kappa_{H}=4 \pi \sigma_{H} /\left(1+4 \pi \sigma_{p o l}\right)$. Eliminating $E_{x}$ and $E_{y}$ from these two equations yields the dispersion relation $\left(\omega^{2}-k^{2} v_{c}^{2}\right)^{2}=\omega^{2} k_{H}^{2}$. For $\omega$ real (appropriate to a boundary-value problem), this dispersion relation can be solved for $k$, glving $k^{2}=\left(\omega^{2} / v_{c}^{2}\right)\left(1 \pm\left(k_{H} / \omega\right)\right)$. When $k_{H}<\omega$ and when propagation toward increasing $z$ is considered, this yields two real solutions for $k$, 


$$
k=\frac{\omega}{v_{c}}\left(1+\frac{\kappa_{H}}{\omega}\right)^{1 / 2}
$$

and

$$
k=\frac{\omega}{v_{c}}\left(1-\frac{k_{H}}{\omega}\right)^{1 / 2},
$$

and when $k_{H}>w$, this ylelds one purely real solution for $k$ and one purely Imaginary solution for $k$,

$$
k=\frac{w}{v_{c}}\left(\frac{k_{H}}{\omega}+1\right)^{1 / 2}
$$

and

$$
k=1 \frac{\omega}{v_{c}}\left(\frac{\kappa_{H}}{\omega}-1\right)^{1 / 2}
$$

In the $k_{H}<\omega$ case (two real-l, solutions), solution ( $32 a$ ) describes a circularly polarized wave with negative helicity, and solution (32b) describes a positive-helicity circularly polarized wave. The phase velocities of the solution ( $32 a$ ) wave (top sign) and the solution (32b) wave (bottom sign) are $v_{p h}=w / k=v_{c}\left[1 \pm\left(\kappa_{H} / w\right)\right]^{-1 / 2}$. Thus, the electron-polarized wave has a higher phase velccity than does the ton-polarized wave. These phase velocities are piottei as Eunctions of $\kappa_{H} / \omega$ in Figure 5 . An initially planepolarized electromagnetic wave is composed of equal parts of solutions (32a) and (32b); as 1t propagates, the plane of polarization will rotate in the sense of the higher-phase-velocity solution. Hence, the electromagnetic wave will have a positive helicity, the plane of polarization rotating in the direction of the electron gyromotion. If the Hall conductivity $\sigma_{H}$ is negative, then the polarization plane rotates in the direction of the ion gyromotion. For $k_{H} \ll \omega$, the plane of polarization of the wave rotates very slowly. As $\mathrm{K}_{\mathrm{H}} \rightarrow \omega$, the plane of polarization rotates through $360^{\circ}$ as the electromagnetlc wave propagates one wavelength (full circular polarization). In this $k_{H}+w$ limit, the positive-helicity solution ( $32 b$ ) ylelds $k \rightarrow 0$ and ${ }^{v_{p h}}+\infty$; hence, there is a cutoff for the positive-helicity solution at $k_{H}=$ 


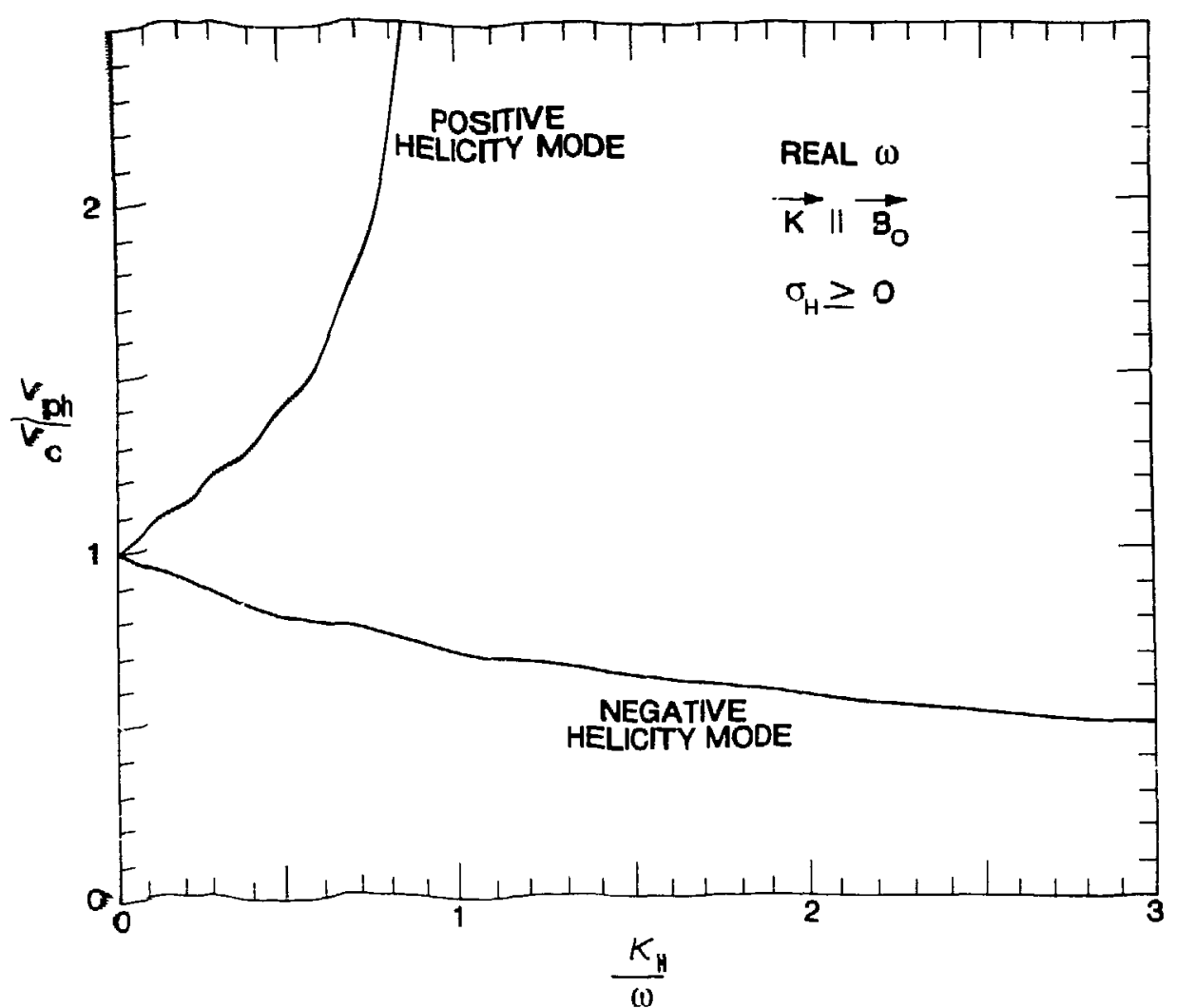

Fig. 5. Appropriate to a boundary-value problein, the phase velocities normalized to $v_{c}=c / \sqrt{1+4 \pi \sigma_{p o l}}$ for the postive-and negativehelectly modes are plotted as functions of the Hall conductivity.

$\omega$, and teflection of that portion of an electromagnetic wav may be expected. For the negative-helicity portion, the solution (32a) is continuous through the $\kappa_{H}=$ wo point (see Figure 5).

For $k_{H}>$ w, propagating (real-k) solution (3ja) is circularly polarized with negative helieity, and nompropagating (imaginary-k) solution (33b) is circularly polarized with positive helicity. The phase velocity of the propagating mode is plotted as a function of $k_{H}$ in Figure 5. Thus, for $\sigma_{H}>$ 0 , in regions where $\mathrm{K}_{\mathrm{H}}>\omega$, the lon-gyromotion-polarized portions of electromagnetc waves are evanescent and the electron-gyromotion-polarized portions propagate without damping. If, instead, the Hall conductivity $\sigma_{H}$ is negative, then $1 \mathrm{t}$ is the electron-polarized portion of an electromagnetic wave that is evanescent.

Note that when equation (31) is solved appropriately for an initial-value problem, It found that both polarizations propagate for all values of $\mathrm{kH}_{\mathrm{H}}$. 
In a collisionless magnetized plasma, the presence of a time-varying, uniform, perpendicular electric fleld alters the gyroperlods of charged particles. When this electric field is increasing in magnitude, the gyroperiods of lons and electrons are increased, and when this fleld is decreasing, the gyroperlods are shortened. For electric fields that vary slowly in time, the gyroperiod of a charged particle may be written $\tau_{c} \simeq$ $\left(2 \pi / \omega c_{c}\right)\left[1 \pm\left(1 / \omega c^{E_{L}}\right)^{2}\left(\partial E_{1} / \partial t\right)^{2}\right]$, where $\omega_{c}=e B_{o} / m c$ is the gyrofrequency in the absence of electric-field time variation. If there are no collisions, then the terms $1 \pi$ the equations of motion ( $A l a$ ) and ( $A l b$ ) that describe this changa do not lead to any drifts, but in the presence of collisions, the time averages of these terms are nonzero. A current in the $\partial \vec{E}_{\perp} / \partial t$ direction arising from the time average of these terms is already accounted for in the polarization conductivity $\sigma_{p o l}$; the current in the $\hat{B}_{0} \times \overrightarrow{\partial E} / \partial t$ direction is described by the cross-polarization conductivity $\sigma_{\text {cpol }}$.

To isolate the cross-polarization effects on fleld propagation, the IImits $\sigma_{\text {ped }} \rightarrow 0, \sigma_{H} \rightarrow 0$, and $\sigma_{\|} \rightarrow \infty$ are taken in constitutive relation ( 5$)$, the combined Faraday (1) and Ampere (2) laws then ylelding the wave equation

$$
\nabla \times 7 \times \vec{E}=-\frac{4 \pi \sigma p o l}{c^{2}} \frac{\partial^{2} \vec{E}_{1}}{\partial \vec{t}^{2}}-\frac{1}{c^{2}} \frac{\partial^{2} \vec{E}}{\partial t^{2}}-\frac{4 \pi \sigma c p o l}{c^{2}} \hat{B}_{0} \times \frac{\partial^{2} \vec{E}}{\partial \vec{E}^{r}} .
$$

The analysis is again simplified by considering only propagation parallel to

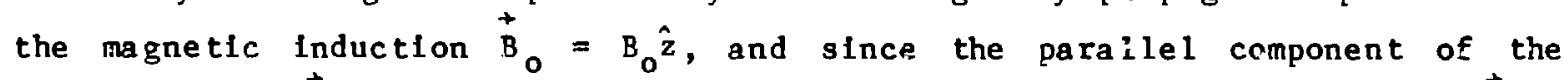
electric fleid $\vec{E}_{\|}$is rot affected by the cross-polarization conductivity, $\vec{E}_{\|}=$ 0 is taken. A normai-mode analysis $e^{1(k z-\omega t)}$ of vector equation (34) yleids the coupled set for the two components of $\vec{E}$ normal to $\vec{B}_{0}$, $\left(\omega^{2}-k^{2} v_{c}^{2}\right) E_{x}=+\omega^{2} \kappa_{c p} E_{y}$ and $\left(\omega^{2}-k^{2} v_{c}^{2}\right) E_{y}=-\omega^{2} \kappa_{c p} E_{x}$, where the dimensionless quantity $\kappa_{c p}$ is defined by $\kappa_{c p}=4 \pi \sigma_{c p o l} /\left(1+4 \pi \sigma_{p o l}\right)$, and where the characteristic velocity $v_{c}$ is again $\pm c / \sqrt{1+4 \pi \sigma}$ pol . Eliminating $E_{x}$ and $E_{y}$ from the coupled set yields the dispersion relation $\left(\omega^{2}-k^{2} v_{c}^{2}\right)^{2}+\omega^{4} \kappa_{c p}^{2}=0$. For w real (approprlate to a boundary-value problem), the dispersion relation ylelds $k^{2}=\omega^{2}(1 \pm 1 k \mathrm{cp}) / v_{c}^{2}$. This rotation has two physical solutions,

$$
k_{\text {real }}= \pm \frac{1}{\sqrt{2}} \frac{\omega}{v_{c}}\left[1+\left(1+\kappa_{c p}^{2}\right)^{1 / 2}\right]^{1 / 2}
$$




$$
k_{\text {Imag }}= \pm \frac{1}{\sqrt{2}} \frac{w}{v_{c}}\left[-1+\left(1+k_{c p}^{2}\right)^{1 / 2}\right]^{1 / 2},
$$

one (the upper signs) corresponding to propagation toward increasing values of $z$ with demping and one (the lower signs) corresponding to propagation toward decreasing $z$ with damping. These solutions represent a positive-helicity and a negative-heilcity component, each having the same phase velocity and damping rate. Thus a plane-polarized wave propagates with damping but does not have its plane of polarization rotated. From equations (35a) and (35b), the phase velocity $v_{\mathrm{ph}}$ and the wavelength to skin-depth ratio $\lambda / \delta$ for an electromagnetic wave are

$$
\begin{aligned}
& v_{p h}+\frac{\omega}{k_{\text {rea } 1}}=\frac{\sqrt{2} v_{c}}{\left[1+\left(1+k_{c p}^{2}\right)^{1 / 2}\right]^{1 / 2}} \\
& \frac{\lambda}{\delta}=2 \pi \frac{k_{1 \text { mag }}}{k_{\text {rea } 1}}=2 \pi \frac{\left[\left(1+k_{c p}^{2}\right)^{1 / 2}-1\right]^{1 / 2}}{\left[\left(1+k_{c p}^{2}\right)^{1 / 2}+1\right]^{1 / 2}} .
\end{aligned}
$$

The phase velocity and the wavelength to skin-depth ratio are plotted as functions of $k_{c p}$ in Figure 6 . As can be seen, the effect of increasing the cross-polarization conductivity is to slow the phase velocity of electromagnetic-wave propagation and to increase the damping of waves.

If the dispersion relation is solved appropricte to an initial-value problem, a similar result is found: propagation with weak damping for both polarizations but with the phase velocity of the disturbance reduced by a factor of $2\left[1+\left(1+\kappa_{c p}^{2}\right)^{-1 / 2}\right]^{1 / 2}$ from that given by relation (36).

\section{INHOMOGENEOUS PLASMAS}

When plasma media are nonhomogeneous, the partial reflection of propagating electromagnetic fields may result. In this investigation, the effects of plasma inhomogeneities are separated into two categories: those caused by changes in the propagation velocity $v_{c}$ and those caused by changes in the conductivities. Changes arise in both categories from changes in the plasma density, in the ambient magnetic induction, or in the collision frequency. The propagation of ulf disturbances in regions of changing 


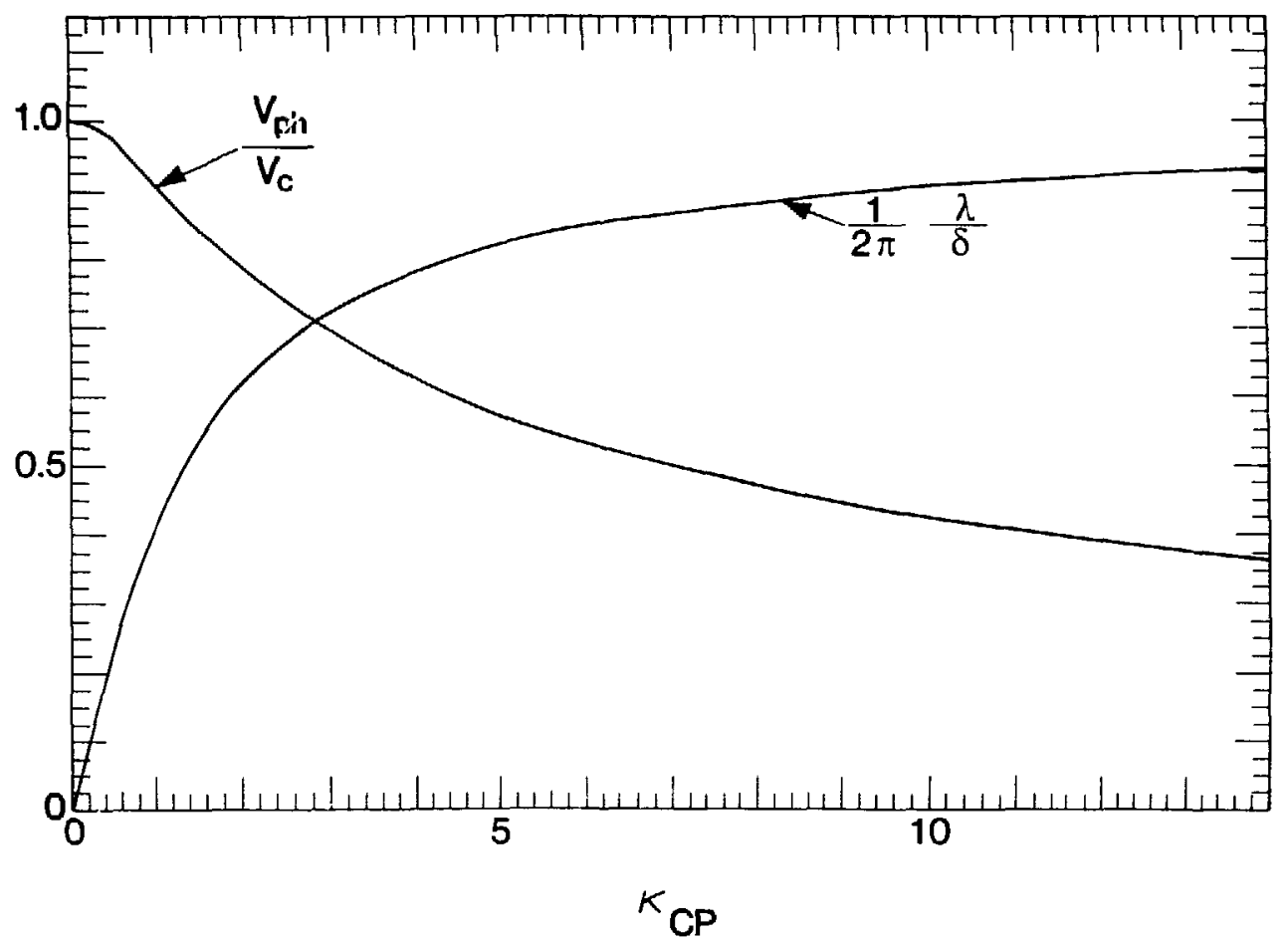

Fig. 6. From equations (36) and (37), the phase velocity $v_{p}$ (normalized to $v_{c}$ $=\sqrt{1+4 \pi \sigma \text { pol }}$ and the wavelength to skin depth for electromagnetic waves are plotted as functions of the cross-polarization conductivity.

characteristic velocity and in regions of changing (ohmic) conductivity is studied separately In Subsections VI.A and VI.B.

\section{A. Inhomogeneous Characteristic Velocity (Density Changes)}

To ascertain the behavior of ultralow-frequency electromagnetic signals propagating in media with spatialiy dependent characteristic velocities, collisionless plasmas with spatially dependent densities are investigated, reflection and transmission coefficients for plasma-density changes being the goal. It will be found that reflection is governed more by the manner with which the density changes than by the amount of change.

For simplicity, wave equation (11) is used with $\overrightarrow{\mathrm{B}}_{0}=\mathrm{B}_{0} \hat{z}$,

$$
\frac{\partial^{2} \vec{E}_{\perp}}{\partial t^{2}}-v_{c}^{2} \frac{\partial^{2} \overrightarrow{E_{1}}}{\partial z^{2}}=0 \text {, }
$$


where the characteristic velocity $v_{c}$ is given by equation (14). Equation (38) describes the guided propagation of a shear-component electric field along $\vec{B}_{0}$ or describes the z-direction propagation of a planar $(\partial / \partial x=\partial / \partial y=0)$ compression-component electric fleld. It is assumed (consistent with the requirements of Section III) that the plasma mass density $\rho=n\left(m_{1}+m_{e}\right)$ and, hence, $v_{A}=B_{0} / \sqrt{4 \pi p}$ a re only functions of $z$. With the assumption thet $\nabla \cdot \vec{E}_{1}$ $=0$, the curl of equation (38) combined with Faraday's law (2) yields, after one time integration, the magnetic-induction wave equation

$$
\frac{\partial^{2} \vec{B}_{\perp}}{\partial z^{2}}-v_{c}^{2} \frac{\partial^{2} \vec{B}_{\perp}}{\partial z^{2}}=2 v_{c} \frac{\partial v_{c}}{\partial z} \frac{\partial \vec{B}_{\perp}}{\partial z}
$$

A comparison of equations (39) and (38) shows that, in regions where $\partial v_{c} / \partial z$ $\neq 0$, the electric field and the magnetic induction do not obey the same wave equation. Among other things, this fact means that the $E$ to $B$ ratio in an inhomogeneous medium will not necessaril, be $v_{c} / c$ (see section 7.1 of Ref, 10). Since mhd-wave reflection and transmission coefficients are usually quoted in terms of $B$ rather than $E$, the analysis here will concentrate on equation (39) rather than on equation (38).

In this subsection, $v_{C}=v_{A}=B_{o} / \sqrt{4 \pi \rho}$ is taken and the propagation of disturbances through linear density ramps is considered. With $v_{C}=v_{A}$, equation (39) becomes

$$
\frac{\partial^{2} B_{\perp}}{\partial t^{2}}-v_{A}^{2} \frac{\partial^{2} B_{\perp}}{\partial z^{2}}+\frac{v_{A}^{2}}{\rho} \frac{\partial \rho}{\partial z} \frac{\partial B_{\perp}}{\partial z}=0 \text {, }
$$

where $\rho$ and $v_{A}(p)$ are functions of $z$. The plasma medium is divided into three spatial reglons; Regions a and $c$ are homogeneous, and Region $b$ is a density ramp connecting Region e to Region $c$. The densities are given by $\rho=\rho_{a}$ for $z$ $<z_{0}, \rho=\rho_{g}+\left(\left(\rho_{c}-\rho_{a}\right) / \Delta x\right)$ for $z_{0}<z<z_{0}+\Delta z$, and $\rho=\rho_{c}$ for $z_{0}+\Delta z<z$.

A numerically simulated truncated-sine-wave pulse propagating through a density ramp with $\rho_{c}=8 \rho_{a}, z_{0}=700$ gridspaces, and $\Delta z=10$ gridspaces is depicted at three instances of time in Figure 7 . In the top panel the incident pulse has not yet reached the ramp; in the middle panel the pulse encounters $i t ;$ and in the bottom panel the reflected and transmitted portions of the pulse are propagating away. Since the density is higher in Region $c$, 

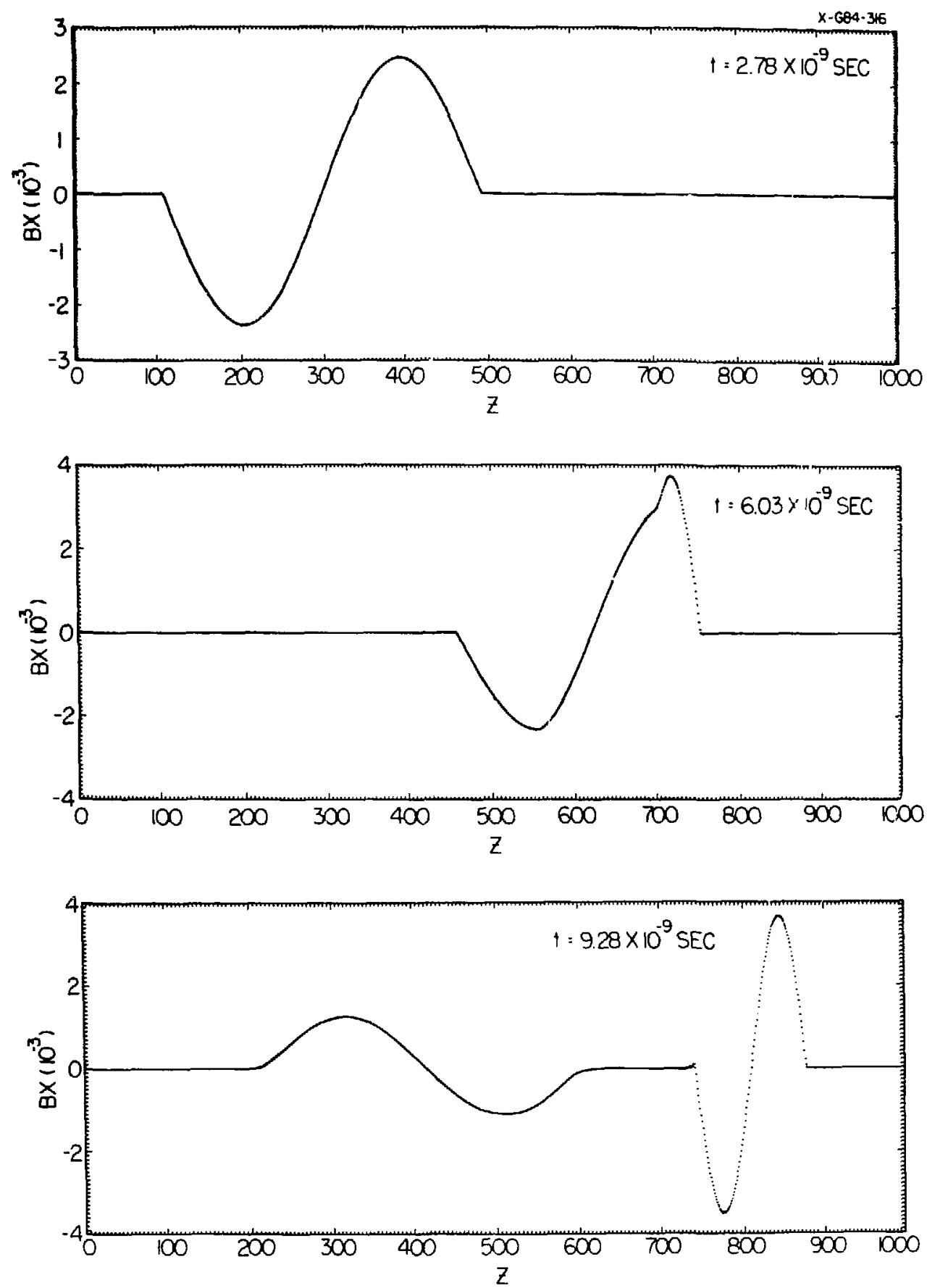

Fig. 7. At three instances of time, a $y=$ constant cut through a pulse propegating into a plasma-density gradient is depicted. 
the Alfven speed is slower there and the pulse wavelength is shorter. Note that the reflected and transmitted pulses have shapes very little distorted from that of the incident pulse. Figure 8 plots the position of the pulse center sa a function of time for the same computer run; the polnts are from the simulation and the lines have slopes equal to the local Alfven speed.

The reflection and trangmission coefficients for such truncated-sine-wave pulses propagating into linear density ramps are plotted as functions of the ramp thickness $\Delta z$ in Figure 9. In each case, the density changes by a factor of 8 across the ramp, $\rho_{c} / \rho_{a}=8$, and in each case, the Incoming-pulse wavelength is 384 gridspaces. The points are from numerical simulations, each reflection-transmission pair from: one computer run, and the curves are from theory. These reflection and transmission coefficients are theoretically obtained in Appendix $C$ by matching solutions to equation (40) across the interfaces between Regions $a$ and $b$ and between Reglons $b$ and $c$, leading to the

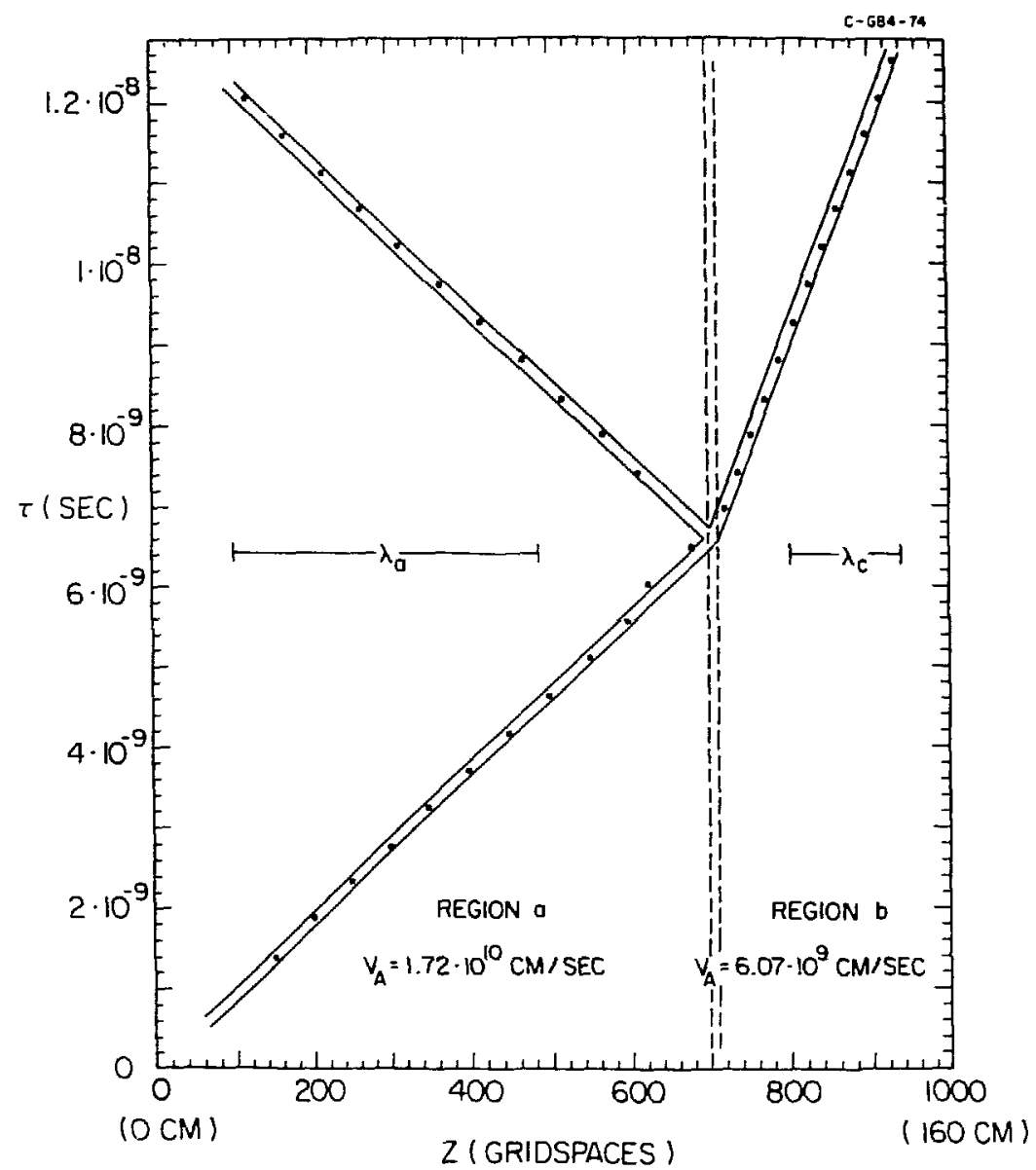

Fig. 8. For the simulation of Figure 7, the position of the pulse center is plotted as a function of time. 


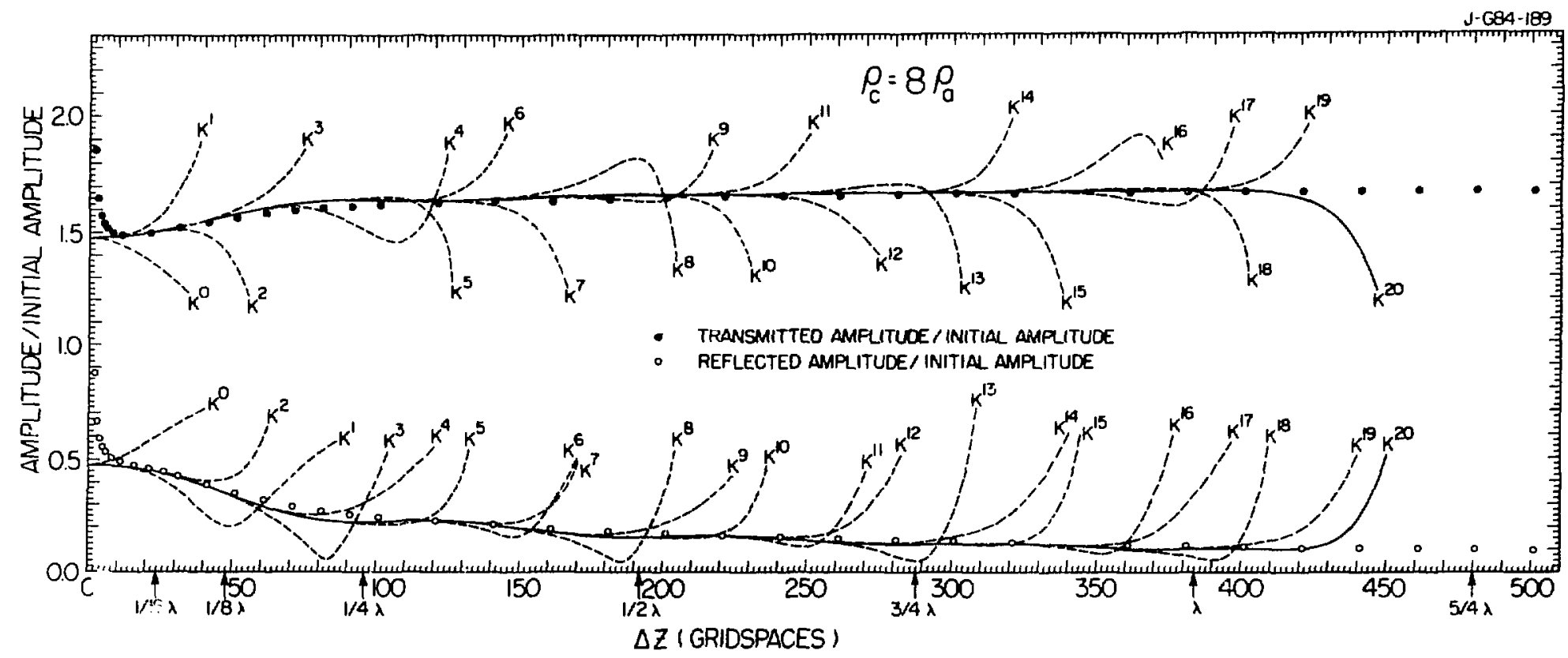

F1g. 9. The reflected and transmitted amplitucies of truncated-sine pulses as functions of $\Delta z$ with $\rho_{c} / \rho_{a}=8$. Points are from simulations, curves from equations ( $\mathrm{C9a}$ ) and $(\mathrm{C} \mathrm{b})$. 
power-serles expressions $(\mathrm{ClOa})$ and $(\mathrm{ClOb})$ for the reflection and transmission coefficlents. These coefficlents are plotted as the curves in Figure 9. The solid curves labeled $K^{20}$ contain all orders up to $n=20$ in expansions ( $C 4 a$ ), $(C 4 b),(C 8 a)$, and ( $C 8 b)$; the dashed curves labeled $k^{n}$ keep terms to order $n$ in these expansions. Although the power-series method converges for all values of $\Delta z / \lambda$, It is very cumbersome for ramp thicknesses $\Delta z$ large compered with the incident wavelengths; as can be seen in Figure 9 , a description of the scattering coefficients for a ramp thickness slightly greater than the incoming wavelength requires retaining 20 or more terms of each of the 4 power series (C4a), (C4b), (C8a), and ( $C 8 b)$. Already, the coefficients of the 20th terms are on the order of $10^{-55}$ to $10^{-57}$, and sums of such numbers are difficult to handle accurately. For ramp thicknesses greater than the pulse wavelengths, approximation methods are necessary.

Falrly good agreement between simulation and theory is found in Figure 9 , except for $\Delta z \leqslant 6$ gridspaces, where the simulations fall badly. This fallure comes about because the $\alpha=1 / 12$ numerical scheme employed to solve the timedependent wave equation (40) uses cubic splines in the $z$-direction, and these splines insure that $B$ and $\partial B / \partial z$ are continuous at each gridpoint. When the scale slzes of density gradients are reduced to the order of the gridspacing, the numerical scheme will keep $a B / \partial z$ constant across these gradients. This condition differs from the boundary conditions taken for the theory of a discontinuity in the medium (see Section 3.4 .5 of Ref. 6); there $B$ and $E$ are continuous across the interface, but $\partial B / \partial z$ is not. For numerical simulations based ufon magnetic-induction equations to be accurate, spatlal changes in the medium must be resolved with more than a few gridpoints. If, Instead, an electric-field equation such as equation ( 38 ) is used, the code accurately treats discontinuous media.

Pulses propagating through Iinear density ramps that have thicknesses $\Delta z$ long compared with the pulse wavelengths are depicted In Figures 10 and 11. The position $z$ of the center of a pulse from five different simulations is plotted as a function from time $t$ in Figure 10 . In each case the piasma medium is unfform with mass densfty $\rho_{a}$ for $0 \leqslant z<150$ gridspaces (Reglon a), is uniform with mass density $\rho_{c}$ for 850 gridspaces < $z=1000$ gridspaces (Region $c$ ), and has a linear density ramp with $\Delta z=700$ gridspaces In-between (Region b). For all five computer runs, the Inftial wavelengths of the pulses originating in Region a are 64 gridspaces, and all runs have the same value of $\rho_{a}$; the differences are in the values of $\rho_{c}$ and the steepnesses of the 


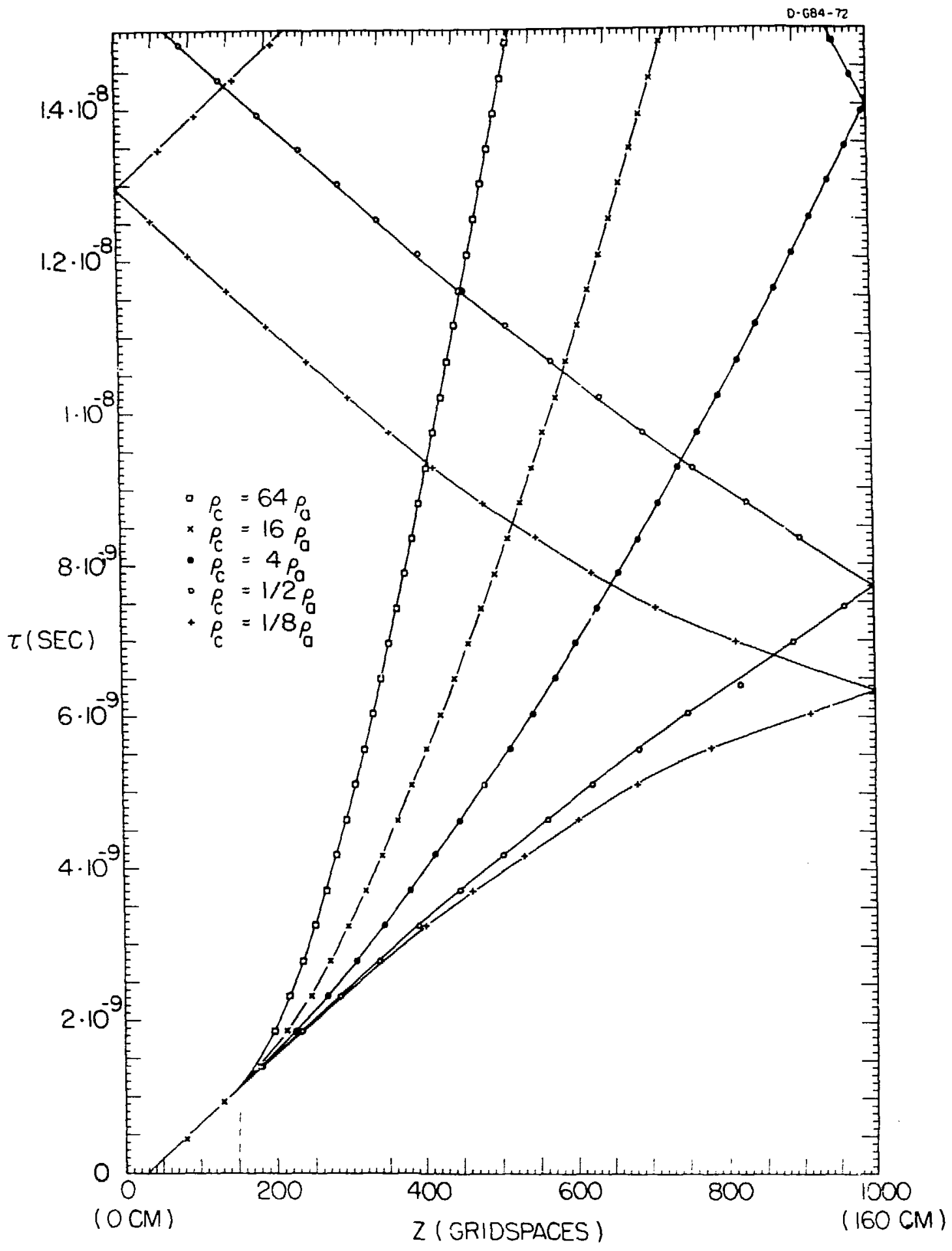

Fig. 10. The position of the centers of pulses propagating into long density ramps with various slopes. The polnts are from simulations and the curves are from theory. 


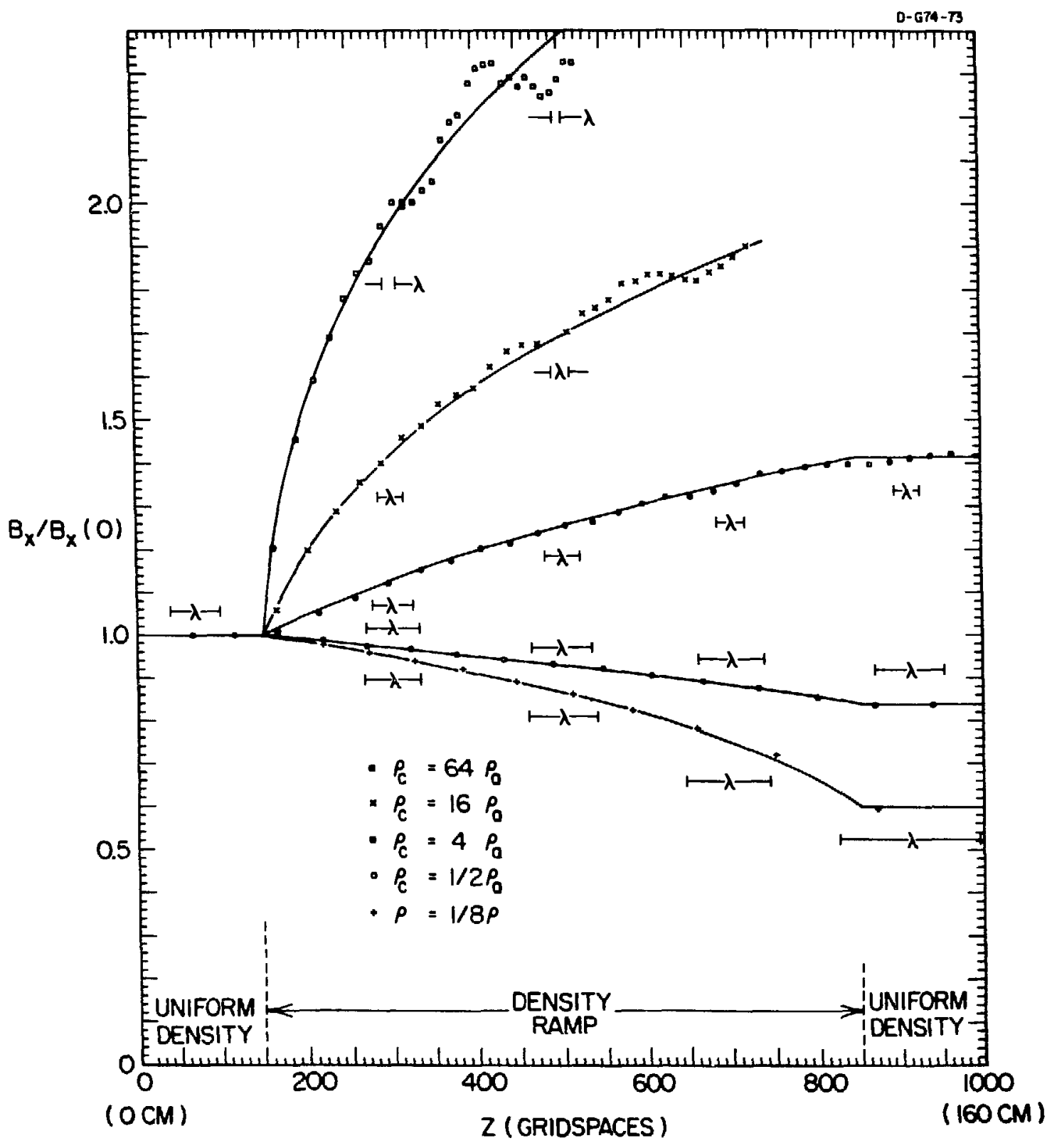

Fig. 1l. For the simulations of Figure 10, the pulse amplitudes are plotted as functions of their positions. The points are from simulations and the curves are from equations (D1) and (D5). 
Region $b$ density ramps. The points are from the five simulations, and the curves are theoretical position-time relations. In Figure 11, the magneticinduction amplitudes of the same pulses are plotted as functions of their positions $z$ within the system. As the pulses travel into regions of Increasing plasma density (top three curves), their velocities decrease, and hence so do their wavelengths. Accordingly, the pulse amplitude Increases, the energy of the pulse being concentrated into a smaller spatial region. These amplitudes are analytically predicted with the approximation method of Appendix $D$, equations (DI) and (D5) appearing as the curves in Figure 11 . The short wavelengths of the pulses within the very high density regions cause the discrepancy for large amplitudes between the numerical simulatioris and the theory; when these wavelengths (Indicated in the figure) become comparable to a few gridspacings, the amplitude of the pulse is difficult to discern accura tely.

In Figure 12, pulse reflection off of a set of linear density ramps with the same slopes $\partial \rho / \partial z$ but with different thicknesses $\Delta z$ is studied by means of numerical simulation. The objective is to determine how deep the incidentreflected pulse penetrates into the ramp. In the top panel, the phase lag of the reflected pulse is plotted as a function of the ramp thickness $\Delta z$, the phase lag being zero if the reflection is off of the interface between Regions a and $b$. The reflected-pulse phase lag is linearly dependent on the ramp thickness $\Delta z$, until $\Delta z \simeq 42$ gridspaces, whereupon the phase lag becomes independent of $\Delta z$. As determined from the phase lag and the velocity of the medium, the maximum depth of penetration into the ramp is plotted as a function of the ramp thickness $\Delta 2$ in the bottom panel of Figure 12 . The Jepth of penetration increases with increasing $\Delta z$ until the depth becomes approximately 17 gridspaces, whereupon it remains constant independent of the ramp thickness and the size of the density change. In each case, the incoming pulse has a wavelength of 384 gridspaces, yet the maximum depth of penetration before the reflected pulse is emitted is 17 gridspaces, only $1 / 22$ of the wavelength. Thus, for $A z>\lambda / 22$, the reflection properties are determined by the foot of the ramp, not by the size of the density change $p_{c} / \rho_{a}$ or by the ramp 

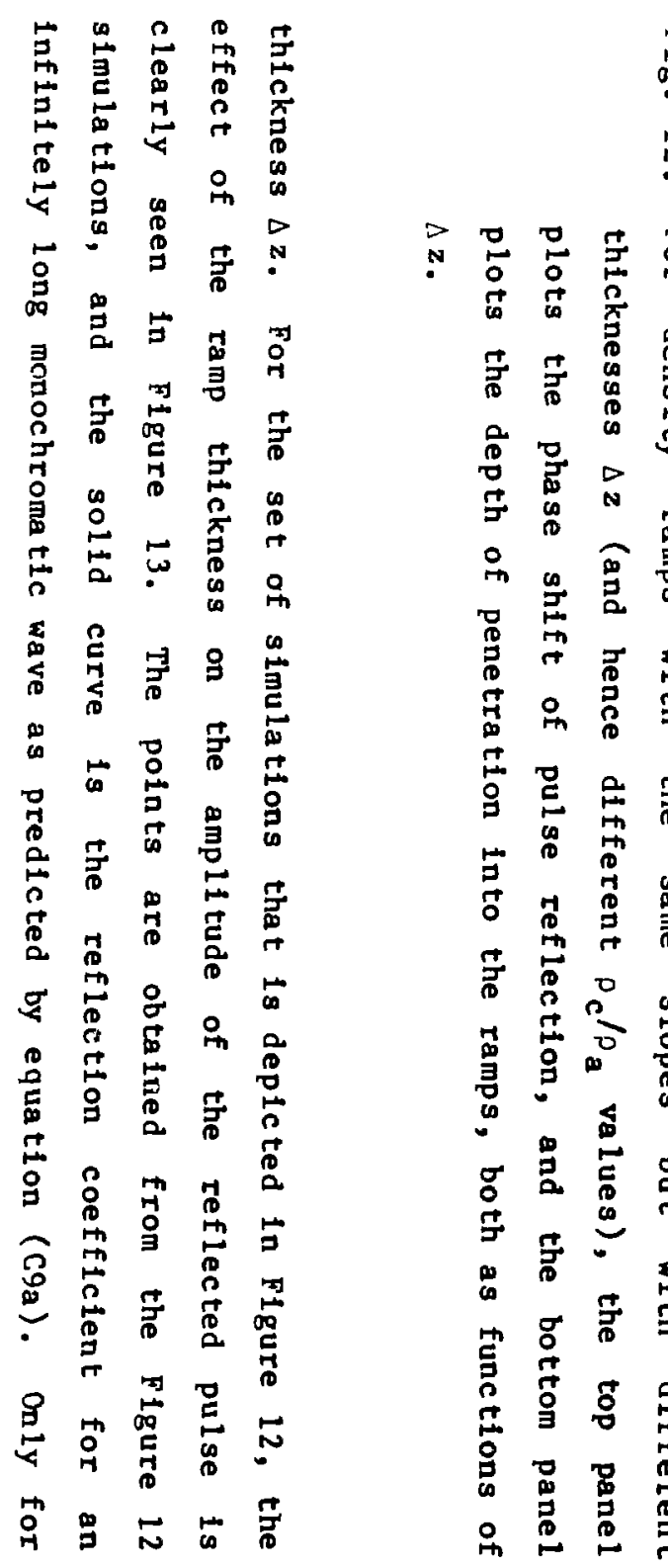

$\underset{9}{9}$

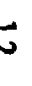

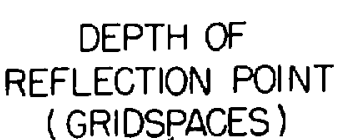

PHASE LAG OF REFLECTED WAVE

( GRIDSPACES)

兽品灾范

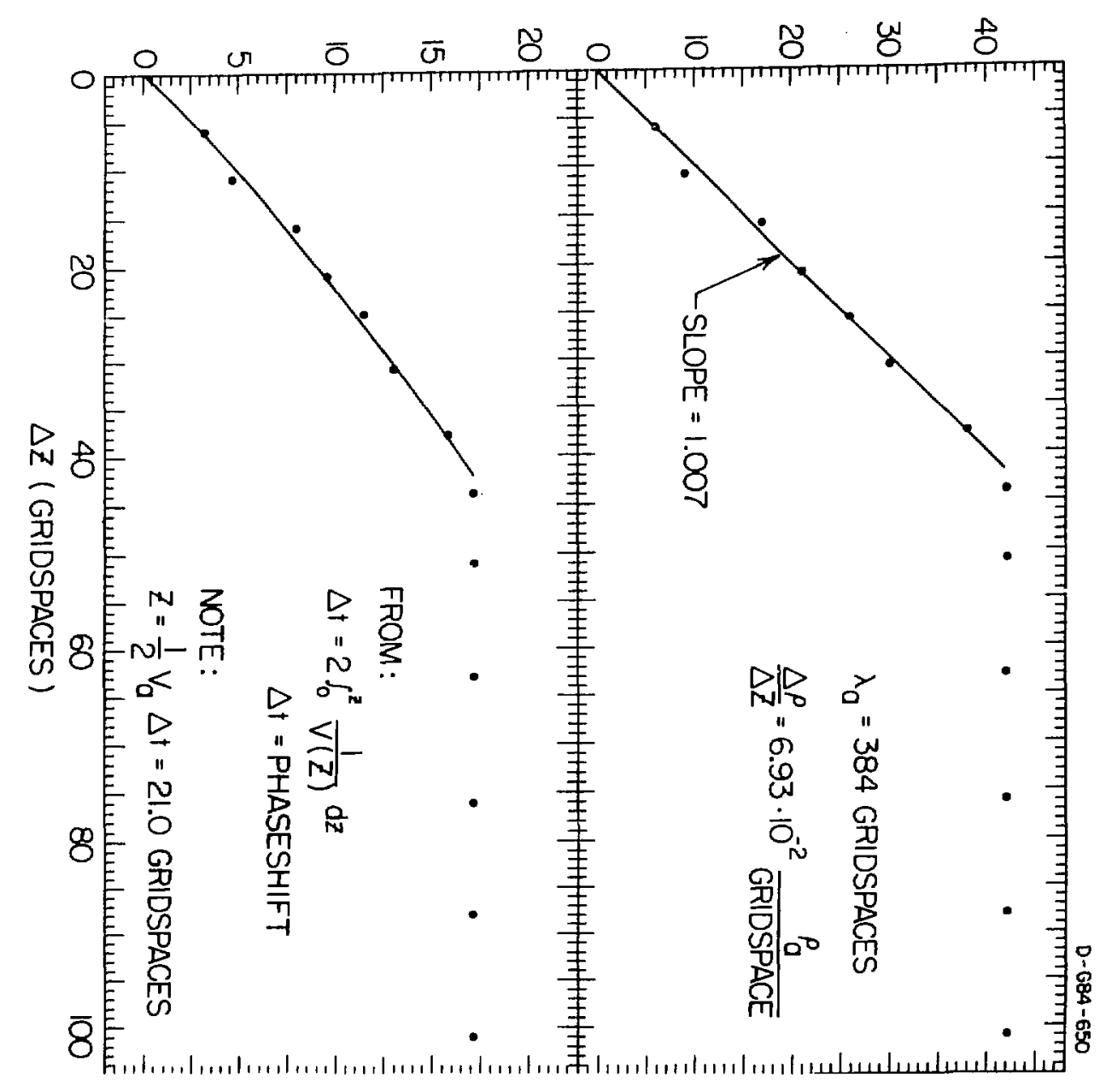




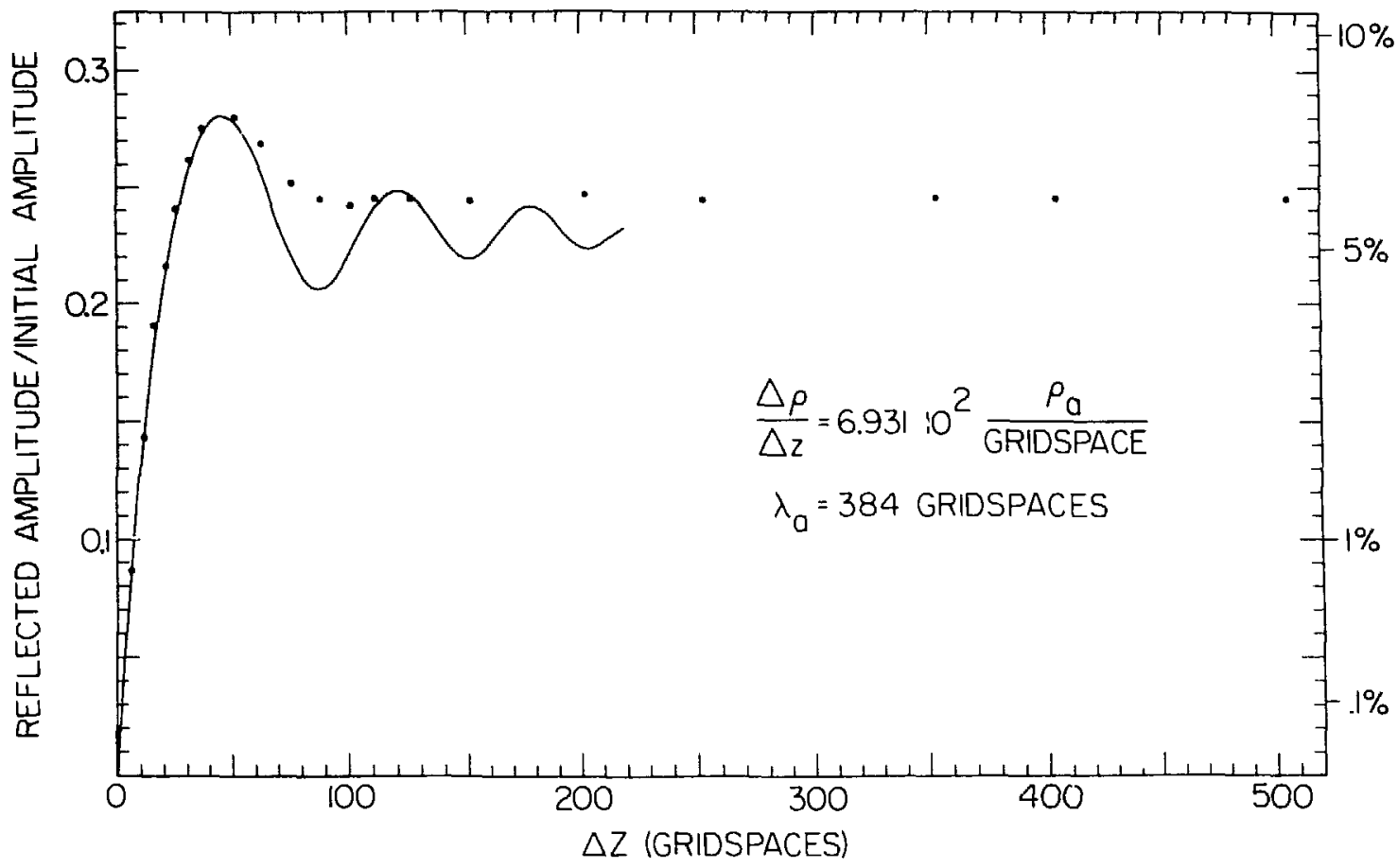

Fig. 13. The amplitude of reflected truncated-sine pulses as a function of $\therefore z$ (same runs as Figure 14). The points are from simulations and the curve is from equation ( $\mathrm{C} 9 \mathrm{a})$.

$\Delta z \leqslant 40$ gridspaces or $\Delta z \leqslant \lambda / 10$ is there a strong dependence of $R$ on $\Delta z$ and, hence, on $\rho_{c} / \rho_{a}$. Density-discontinuity calculations for the reflection and transmission coefficients [as in equations ( $C 10 a$ ) and ( $C 10 b)$ ] will fail badly unless $\lambda$ is very much greater than $\Delta z$; the coefficients given by those calculations depend only on $\rho_{c} / \rho_{a}$, whereas the actual coefficients may be independent of $\rho_{c} / \rho_{a}$. In general, the steeper the density gradient is, the smaller the penetration depth and the more important the shape of the density profile. These results indicate that the reflection and transmission of ulf electromagnetic waves off of such plasmas as the terrestrial ionosphere will be very sensitive to the plasma profile. Care must be exercised in modeling such systems because breakpoints in analytical fits may produce artificial reflections. 
In this subsection, the propagation of low-frequency electromagnetic Iisturbances in plasmas with inhomogeneous conductivities is studied by using an electric-field equation. For shear-polarization fields in media with spatially dependent Pederson or parallel conductivities [equations (22) or (30)] or for compressive-polarization fields in media with spatially dependent Pederson conductivities [equation (18a)], equations of the form

$$
\frac{\partial^{2} E}{\partial t^{2}}-v_{c}^{2} \frac{\partial^{2} E}{\partial z^{2}}=\kappa(z) \frac{\partial E}{\partial t}
$$

apply. Simulations of truncated-sine wave trains propagating through linear conductivity ramps are performed, and the results are compared with reflection coefficients obtained from impedance-matching calculations. Airy-function expansions (as developed in Appendix C) for linear conductivity ramps are cumbersome and do not converge well, so they are not used.

A planar wave train after encountering a linear conductivity $r a m p$ is depicted in Figure 14. The conductivity is zero on the left-hand side of the figure ( $z<0.42$ ), and then it linearly ramps up over 10 gridspaces to a inite value. Initially, the wave train was propagating in the zero-conductivity region toward the conductivity ramp. In the figure the wave train has encounterd the ramp; a reflected signal is propagating in the zero-conductivity region, a transmitted signal is propagating in the finiteconductivity region, and the transmitted signal is damping exponentially with distance as it propagates because of ohmic loss.

Figure 15 was compiled from 84 computer runs of wave trains propagating from collisionless plasmas into linear conductivity ramps matched onto plasmas with finite and homogeneous conductivities. The figure plots the electric- or magnetic-field-amplitude reflection coefficient $R$ as $a$ function of the conductivity of the collisional plasma. Four different ramp thicknesses $\Delta z$ are used, and in all runs the wavelength within the wave train is $\lambda=96 \mathrm{grid}-$ spaces. Clearly discernible is the fact that the reflection coefficient is higher if the conductivity value across the ramp is higher or if the ramp is thinner. Plotted as the solid curve in Figure 15 is the reflection coefficient (Ela) predicted from an impedance-matching calculation in Appendix E. The impedance-matching prediction is a poor fit to the finite-rampthickness simulations, the impedance-matching prediction overestimating the reflected amplitude. 


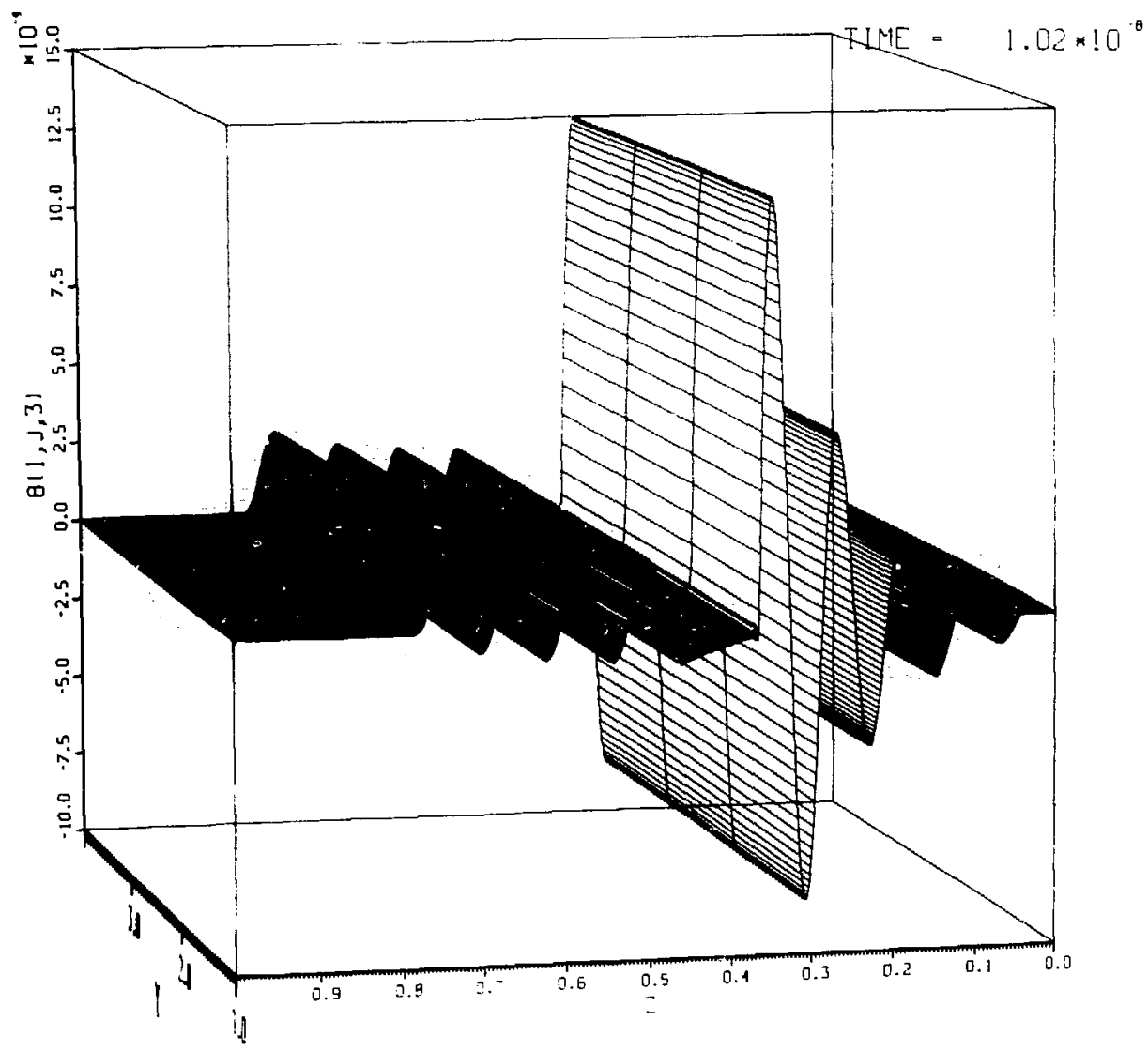

Fig. 14. For planar wave train that propagated into a conductivity ramp, the resulting reflected and transmitted trains are deplcted propagating away from the ramp.

These coefficients are more visible in Figure 16, which was composed from 50 computer runs of truncated-sine wave trains propagating into linear conductivity ramps. The electric-field reflection coefflclent $R$ is plotted as a function of the ramp thickness $\Delta z$ for four values of the ramp slope $\partial \sigma / \partial z$. The value of the conductivity on the transmission side of the ramp is thus $\sigma=$ $(\partial \sigma / \partial z) \Delta z$, with $\sigma=0$ on the incidence side. In each computer run, the wavelength is $\lambda=400$ gridspaces within the wave train. Each point plotted is the value obtained from one simulation, and the solid curves are obtalned from equation (Ela). Here, it is seen that whenever the reflectance of the ramp is high ( $R$ near 1.0 ) the impedance-matching calculation overestimates $R$. That 


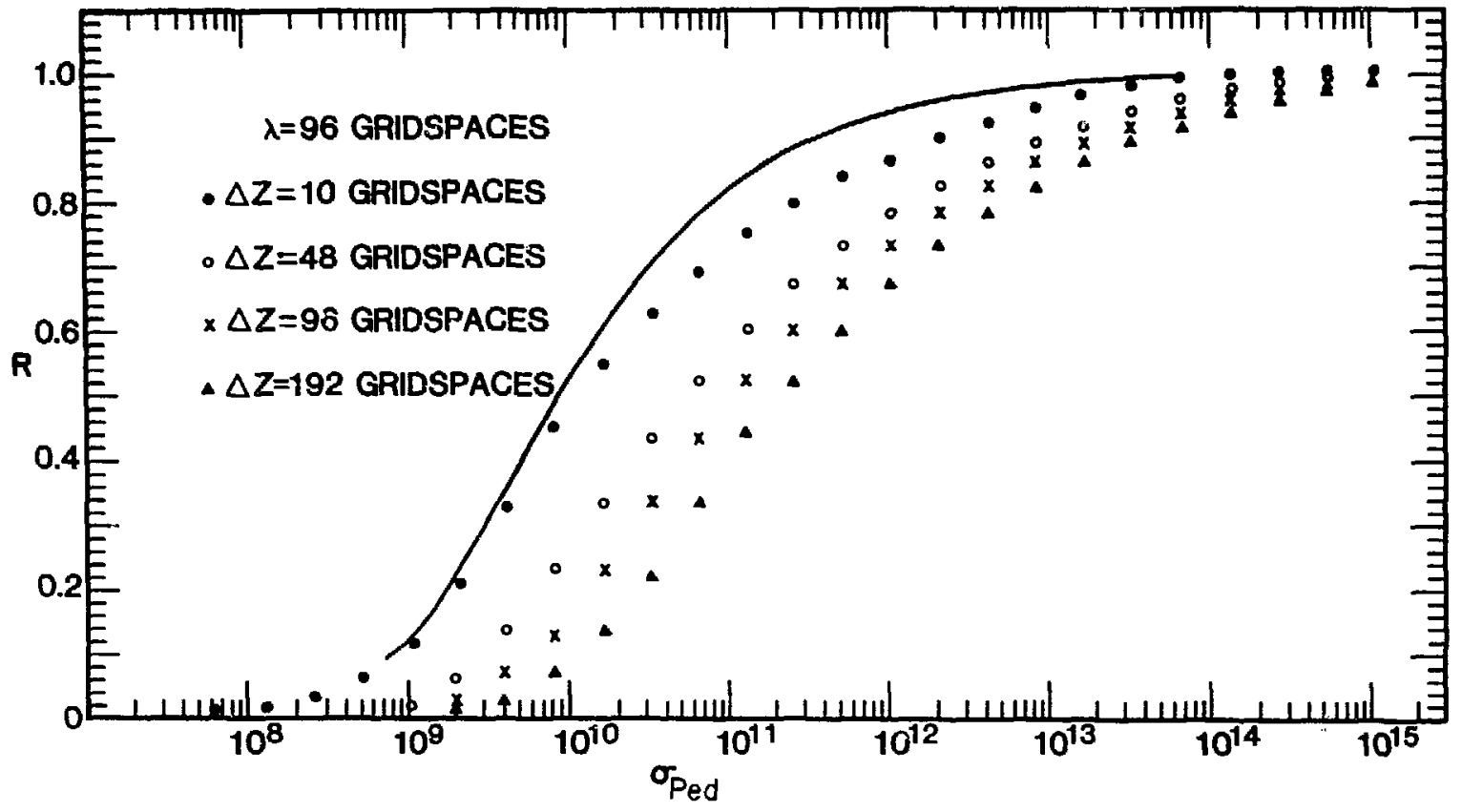

Fig. 15. The reflection coefficient for wave trains propagating from collisionless plasmas into collisional plasmas is plotted as a function of the Pederson conductivity of the collisional plasma. The points are from simulations (with various transition-layer thicknesses) and the curve is from equation (Ela).

1s, when $R$ is high, the fraction of wave energy that is not reflected is significantly larger than the impedance matching would indicate. For these discontinuity calculations to be accurate for $R$ near unity, the thickness of the conductivity ramp must be much smaller than that of the incident wavelength $(\Delta z<\lambda / 80$ at $R=0.9)$ and the conductivity jump must be very large. As can be seen in Figure 16, a fter $\Delta z$ reaches a certain size, the reflection coefficient becomes independent of $\Delta z$. The conductivity jump being proportional to $\Delta z$, the actual reflection coefficient becomes independent of $\sigma$, whereas the impedance matching has $R$ depending only on $\sigma$.

As was the case for density ramps, the reflection and transmission coefficients for coaductivity ramps are very sensitive to the ramp profile, slight modifications to the foot of a conductivity ramp sometimes resulting in substantial modifications to the reflection coefficient. 


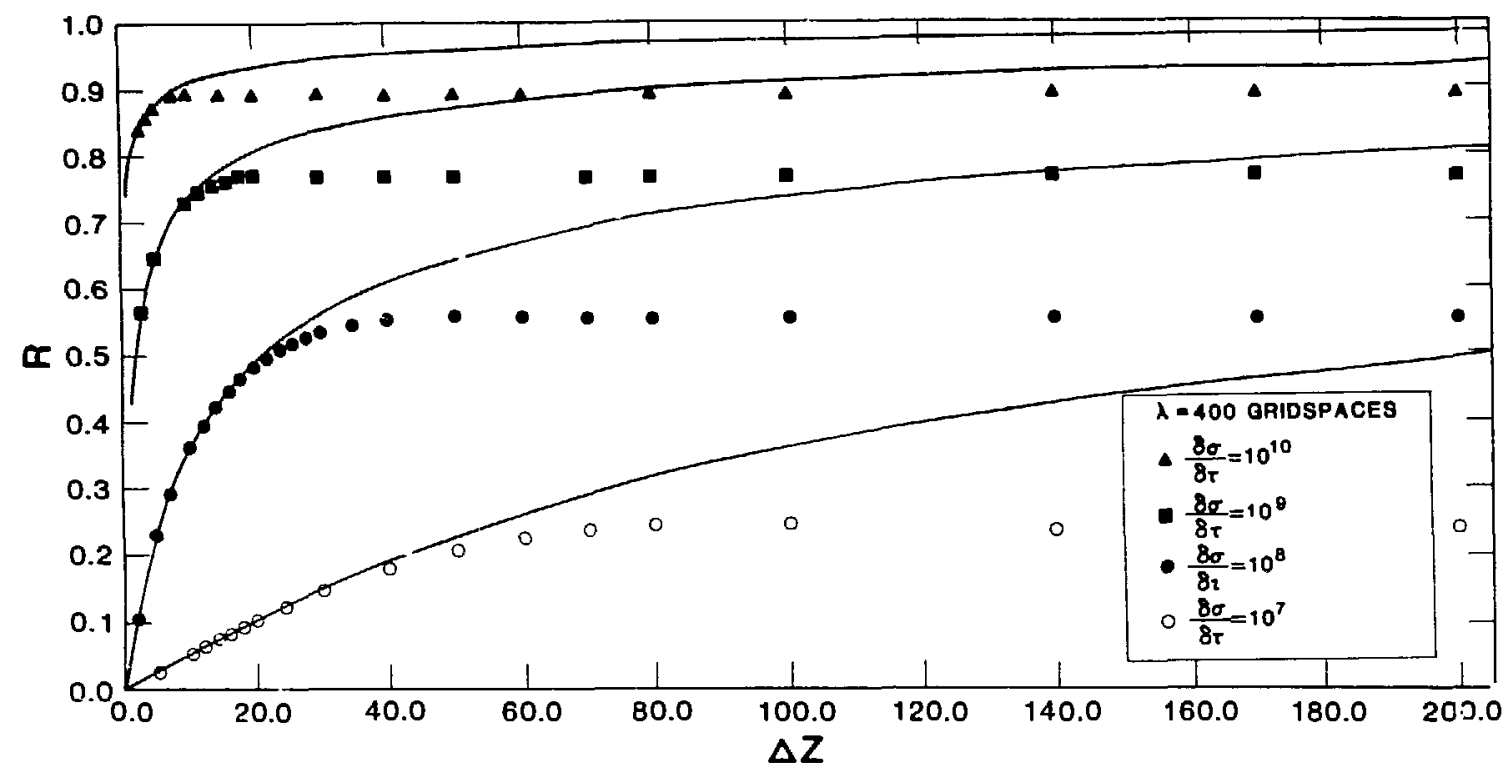

Fig. 16. The reflection coefficient for wave trains propagating from collisionless plasmas into collisional plasmas is plotted as a function of the transition-layer thickness. The points are from simulations, and the curves are obtained from equation (Ela) by using the fact that $\sigma_{\operatorname{Ped}_{1}}=0$ and $\sigma_{\operatorname{Ped}_{2}}=\left(\partial \sigma_{\text {ped }} / \partial z\right) \Delta z$.

VI. FUTURE PROBLEMS

This report describes some of the results obtained from a computer code ${ }^{2}$ that was developed to study the propagation and damping of $\omega<\omega_{c i}$ electromagnetic fields in collisional or collisionless inhomogeneous plasmas. In this section, some of the research topics to which this code will be applied are described.

The reflection, transmission, and absorption coefficients for lowfrequency $(<0.1 \mathrm{~Hz}$ ) electromagnetic disturbances propagating from the terrestrlal magnetosphere toward the Earth will be cbtained by the use of numerical simulations. Careful modeling of the lonospheric plasma density is necessary to obtain the correct coericients; matching analytical approximations from one altitude region to another may produce spurious reflections. These coefficients are pertinent to the magnetosphere/ionosphere coupling in the auroral zone and to the predictions of ground signals arising from geomagnetic disturbances. 
The reflection, transmission, and attenuatlon coefficients will also ve obtained for the propagation of electromagnetic flelds from the ground into the terrestrial magnetosphere. These coefficients are relevant to the coupling of $50-$ and $60-\mathrm{Hz}$ power-line radiation to space and may provide a test for the simulations by comparison of numerical predictions with measurements of ulf signals generated from ground-based milltary antennae.

As the conducting moon Io orbits Juplter, it strongly perturbs the Jovian magnetospheric plasma. Some of this disturbance propagates as a low-frequency shear-polarization fleld. The shear-polarization disturbance is of interest because its propagation is guided along the Jovian magnetic field and energy from the motion of Io can be transported onto a small spot on the lonosphere. The ability of these waves to escape from the dense torus plasma into the tenuous magnetospheric plasma will be studied, and the reflection, transmission, and absorption coefficlents for these waves on the Jovian Ionosphere will be obtained.

The propagation of low-frequency electromagnetic waves through the solar a tmosphere/corona will also be numerically simulated, the damping rates and transmission coefficients as functions of the wave frequency $\omega$ being the goal. These values are pertinent to the estimation of energy transport from the solar surface to the solar wind.

The propagation of shear-mode disturbances in neutron-star magnetospheres will be numericaliy simulated to determine the reflectivity of accretion disks and neutron-star surfaces. Such reflection coefficients are pertinent to theories concerning the establishment of electrical circuits that couple the various regions of the neutron-star magnetospheres.

\section{ACKNŌWLEDGMENTS}

The author wishes to thank Christoph Goertz and Robert Roussel-Dupre for extended conversetlons and wishes to thank Glenn Joyce, Morris Pongratz, and Karla Sofaly for their ald. 
The conductivities for low-frequency phenomena in magnetized collisional plasmas are derived from mean-free-path theory as follows. The curcent density $\vec{J}$ is given by the difference between the time-averaged $d r i f t$ velocities of a typical ion and a typical electron, $\vec{j}=n_{0} e\left(\left\langle\vec{v}_{1}\right\rangle-\left\langle\vec{v}_{e}\right\rangle\right)$, where $\Longleftrightarrow$ denotes averaging over time. Between collisions, the orbit of a particle of mass $m$ and charge $q$ within a magnetic induction $\vec{B}_{0}$ is determined. by the equation of motion $\vec{a} \vec{v}(t) / a t=(q / m)\left[\vec{E}(t)+\left(\vec{v}(t) \times \vec{B}_{0}\right) / c\right]$, where the Lorentz force arising from the perturbation magnetic induction $\vec{B}(t)$ is ignored. It is assumed that an orbiting particle sees a perturbation electric field $\vec{E}(t)$ that is spatially homogeneous; this requires the particles to move at velocities slow compared with the fleld-propagation velocities. In the equation of motion, the parallel motion is not written as a time integral of the electric fleld; this is equivalent to requiring parallel-moving particles to suffer momentum-exchange collisions frequently compared with the timescale of the electromagnetic disturbance. For $\vec{B}_{0}=B_{0} \hat{z}$, to first order in $\left(1 / \omega_{c}\right)(\partial / \partial t)$, the equation of motion leads to the orbit equations

$$
\begin{aligned}
& v_{x}(t)=v_{x_{0}} \cos \left(Q_{\omega_{c}} t\right)+v_{y o} \sin \left(Q_{\omega_{c}} t\right) \\
& +\left[\frac{c}{B_{0}} E_{y}(t)+\frac{m c^{2}}{q B_{0}^{2}} \frac{\partial E_{x}}{\partial t}\right]\left[1-\cos \left(Q_{c} t\right)\right] \\
& +\left[\frac{c}{B_{0}} E_{x}(t)-\frac{m c^{2}}{q B_{0}^{2}} \frac{\partial E_{y}}{\partial t}\right] \sin \left(Q_{w} t\right) \\
& v_{y}(t)=v_{y_{0}} \cos \left(Q_{\omega_{c}} t\right)-v_{x_{0}} \sin \left(Q_{\omega_{c}} t\right) \\
& +\left[-\frac{c}{\bar{B}_{0}} E_{x}(t)+\frac{m c^{2}}{q B_{0}^{2}} \frac{\partial E_{y}}{\partial t}\right]\left[1-\cos \left(Q_{c} t\right)\right] \\
& +\left[\frac{c}{B_{0}} E_{y}(t)+\frac{m c^{2}}{Q B_{0}^{2}} \frac{\partial E_{x}}{\partial t}\right] \sin \left(Q_{c} t\right) \\
& v_{z}(t)=\frac{q}{m} E_{z}(t) t+v_{z 0},
\end{aligned}
$$


where $Q=q /|q|$. Note tr. equation ( $A l c$ ) is accurate only for times that are short compared with the electric-field variation. The orbit equations (Ala) (Alc) describe gyromotion, $\vec{E} \times \vec{B}$ drifting, polarization drifting, and parallel acce le ra tion.

Following Townsendll, 12 it is assumed here that momentum-transfer collisions are distributed in time according to $f=v e^{-v t}$, where $\nu$ is the momentum-transfer collision frequency; this being the case, the time-averaged velocity of a charged particle is given by

$$
\overrightarrow{\langle v\rangle}=\int_{0}^{\infty} \vec{v}_{\text {orbit }} \nu e^{-v t} d t \text {, }
$$

where $\vec{v}_{\text {orbit }}$ is the colilisionless trajectory given by equations (Ala) - (Alc). By definition, momentum-transfer collisions leave a particle with an average velocity of zero after one collision time; thus, $v_{x o}=v_{y o}=v_{z o}$ is taken in equations (Ala) - (A1c). Inserting these orbits into relation (A2) yields

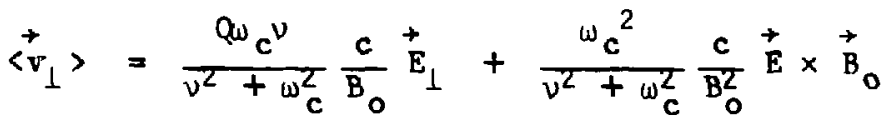

$$
\begin{aligned}
& +\frac{\omega_{c}^{2}}{v^{2}+\omega_{c}^{2}} \frac{m c^{2}}{\mathrm{~B}^{2}} \frac{\partial \overrightarrow{\mathrm{E}}_{0}}{\partial t}+\frac{Q_{\omega} c^{\nu}}{v^{2}+\omega_{c}^{2}} \frac{m c^{2}}{\mathrm{qB}_{0}^{2}} \hat{B}_{0} \times \frac{\partial \overrightarrow{\mathrm{E}}}{\partial t}
\end{aligned}
$$

and

$$
\left\langle\mathbf{v}_{\| 1}\right\rangle=\frac{q}{\mathrm{~m}} \frac{1}{v} \mathrm{E}_{\eta}
$$

By inserting relations (A3) for lons and for electrons into $\overrightarrow{\mathrm{J}}=$ $n_{0} e\left(\left\langle\vec{v}_{1}\right\rangle-\left\langle\vec{v}_{e}\right\rangle\right)$, the current density can be written in the form

$$
\vec{J}=\sigma_{p e d} \vec{E}_{\perp}+\sigma_{\|} \vec{E}_{\|}+\sigma_{p o 1} \frac{\partial E_{\perp}}{\partial t}+\sigma_{H} \hat{B}_{0} \times \vec{E}+\sigma_{c p o l} \hat{B}_{0} \times \frac{\partial E}{\partial t},
$$

where the various conductivities o are written as equations (7a) - (7e). 
Equations of the form.

$$
\frac{\partial^{2} \mathrm{f}}{\partial t^{2}}-v_{c}^{2} \frac{\partial^{2} \mathrm{f}}{\partial z^{2}}=-k \frac{\partial \mathrm{f}}{\partial t}
$$

occur of ten when electromagnetic propagation with ohmic disatpation $\vec{J} \propto \vec{E}$ is considered, as in the propagation of compressive (magnetosonic) disturbances In media with finite $\sigma$ ped [equation (18a)], in the propagation of shear (Alfvenic) disturbances in media with finite $\sigma_{\text {ped }}$ [equation (22)] or finite $\sigma_{\|}$ [equation (30)], or in the propagation of ordinary-mode disturbances in media with finite $\sigma_{\|}$[equation $(18 b)$ ]. Equation (BI) is the equation of telegraphy, first discussed for electromagnetic-field propagation by Maxwell.8 It is hyperbolic, with characteristic velocity $d z / d t= \pm v_{c}$, and dissipative for $k>$ 0 .

If the medium of propagation is homogeneous and time independent, then $v_{c}$ and $k$ are not functions of $z$ or $t$, and equation (B1) may be normal-mode analyzed $e^{1(k z-\omega t)}$ to yleld the dispersion relation

$$
\omega^{2}+1 k \omega-k^{2} v_{c}^{2}=0
$$

This dispersion relation may be solved for $\omega$ with $k$ real (appropriate to an inftial-value problem) or solved for $k$ with $w$ real (appropriate to a boundaryvalue problem).

For $k$ real and given (Initial-value problem), relation (B2) yields

$$
\omega= \pm\left\lceil k^{2} v_{c}^{2}-\frac{k^{2}}{4}\right\}^{l / 2}-1 \frac{k}{2}
$$

If $k^{2}<4 k^{2} v_{c}^{2}$, then this equation has the solutions 


$$
\begin{aligned}
& w_{\text {real }}= \pm v_{c}\left[1-\frac{k^{2}}{4 k^{2} v_{c}^{2}}\right]^{1 / 2} \\
& w_{\text {imag }}=-\frac{k}{2} \\
& v_{\text {ph }}+\frac{\omega_{\text {rea }}}{k}= \pm v_{c}\left[1-\frac{k^{2}}{4 k^{2} v_{c}^{2}}\right]^{1 / 2}
\end{aligned}
$$

and a disturbance propagates with finite velocity, and its amplitude is damped to $l / e$ of 1 ts value in a time equal fo $2 / \mathrm{K}$. The finite-k medium is dispersive, the phase velocity $v_{p h}$ (which goes to $\omega_{r e a l} / k$ as time goes to $\infty$ ) being faster for the higher-k components of a ilsturbance. If $k^{2}>4 k^{2} v_{c}^{2}$, then Initial-value disturbances no longer propagate, the telegrapher's equation being in a diffusion-like regime. In this case relation (Bj) gives

$$
\begin{aligned}
& { }^{\omega} \text { rea } 1=0 \\
& { }_{1 \mathrm{Imag}}=\frac{k}{2}\left[1 \pm\left(1-\frac{4 k^{2} v_{c}^{2}}{k^{2}}\right)^{1 / 2}\right] \\
& v_{p h}+\frac{w_{\text {real }}}{k}=0 \text {, }
\end{aligned}
$$

a stationary disturbance damping away exponentially with time. Note that for $k+0$, there is never propagation, even if $k$ is very small. For $w$ real and given (boundary-value problem), relation (B2) yields the solutions

$$
\begin{array}{ll}
k_{\text {rea } 1} & = \pm \frac{1}{\sqrt{2}} \frac{\omega}{v_{c}}\left[\left(1+\frac{\kappa^{2}}{\omega^{2}}\right)^{1 / 2}+1\right]^{1 / 2} \\
k_{\text {mag }} & = \pm \frac{1}{\sqrt{2}} \frac{\omega}{v_{c}}\left[\left(1+\frac{\kappa^{2}}{\omega^{2}}\right)^{1 / 2}-1\right]^{1 / 2}
\end{array}
$$




$$
v_{p h}+\frac{\omega}{k_{\text {rea } 1}}=\sqrt{2} v_{c}\left[\left(1+\frac{k^{2}}{\omega^{2}}\right)^{1 / 2}+1\right]^{1 / 2},
$$

$k_{\text {imag }}$ being the absorption coefficient. The attenuation length or skin depth $\delta$ is defined as the distance that the wave penetrates into the plasma before its amplitude is attenua ted by $1 / \mathrm{e}$,

$$
\delta=\frac{1}{k_{\text {imag }}}=\sqrt{2} \frac{v_{c}}{\omega}\left[\left(1+\frac{\kappa^{2}}{\omega^{2}}\right)^{1 / 2}-1\right]^{-1 / 2} .
$$

By ana logy with mhd (see Section 3.9 of Ref. 6), a Lundquist number $L$ is defined as a measure of the importance of the damping term $\kappa(\partial f / \partial t)$ in equation (B1),

$$
L=\frac{k v_{c}}{K} \text {, }
$$

where $k=2 \pi / \lambda, \lambda$ being a measure of the size of a disturbance. According to definition ( $B 8$ ), the crossover from propagation to no propagation for an Intial-value problem occurs at $L=1 / 2$. 
For $v_{A} \ll c$, the propagation of planar ulf electromagnecic disturbances is described by equation (40). In this Appendix, the reflection and transmission coefficients for propagation through linear ramps in the plasma density are obtained by matching power-series solutions of equation (40) across the interfaces between Regions $a$ and $b$ and between Regions $b$ and $c$ (In the notation of Section VI.A).

To obtain solutions in Region b, equation (40) is split into a spatial and a temporal piece by assuming that solutions are of the form $B_{1}(z, t)=$ $f(z, \omega) e^{-f \omega t}$; with the substitution $\zeta=1+\left[\left(p_{c}-p_{a}\right) / p_{a}\right]\left[\left(z-z_{0}\right) / \Delta z\right]$, the Region $b$ time-independent wave equation becomes

$$
\zeta \frac{\partial^{2} f_{b}}{\partial \zeta^{2}}-\frac{\partial f_{b}}{\partial \zeta}+K \zeta^{2} f_{b}=0
$$

where the constant $K=\left[\omega^{2} / v_{A a}^{2}\right]\left[\rho_{a}^{2} \Delta z^{2} /\left(\rho_{c}-\rho_{a}\right)^{2}\right]-1$ and where $\Delta z$ is the ramp thickness. Writing $f_{b}$ in the form of a power series,

$$
f_{b}(\zeta, \omega)=\sum_{n=0}^{\infty} a_{n}(\omega) \zeta^{n}
$$

equation ( $\mathrm{Cl}$ ) becomes

$$
\sum_{n=2}^{\infty}\left[(n+1)(n-1) a_{n+1}+K a_{n-2}\right] \zeta^{n}-a_{1}=0 \text {. }
$$

Equating equal powers of $\zeta$, equation (C3) ylelds the coefficients $a_{n}$ to give the Region bolution $f(\zeta)=a_{0} s_{1}+a_{1} s_{2}$, where

$$
\begin{aligned}
& s_{1}(\zeta)=1+\sum_{n=1}^{\infty} \frac{(-K)^{n}}{(3 n) !}\left[\prod_{m=1}^{n}(3 m-1)\right] \zeta^{3 n} \\
& \left.s_{2}(\zeta)=\zeta^{2}=2 \sum_{n=1}^{\infty} \frac{(-K)^{n}}{(3 n+2) !} \prod_{m=1}^{n}(3 m+1)\right] \zeta^{3 n+2} .
\end{aligned}
$$


The series ( $\mathrm{C} 4 \mathrm{a}$ ) and ( $\mathrm{C} 4 \mathrm{~b})$ both converge for any value of $\mathrm{K}^{3}{ }^{3}$. The mathematical nature of the solution $f_{b}(\omega, z)$ to the time-independent wave equation ( $\mathrm{Cl}$ ) can be. discerned with the change of variables $\gamma=$ $k^{1 / 3}\left(1+z\left[\left(\rho_{c}-\rho_{a}\right) / \rho a^{\Delta z}\right]\right)$ and $f(\gamma)=\int^{\gamma} \gamma^{-} A_{i}\left(\gamma^{-}\right) d \gamma^{-}$. With these new quantities, equation (Cl) becomes (after one differentiation) $\partial^{2} A_{1} / \partial \gamma^{2}+\gamma A_{i}=$ 0 , which is Alry's equation (equation 10.4.1 of Ref. 13). Thus solutions to equation (C1) in a linear density ramp are

$$
f_{b}(z)=k^{2 / 3} P \int^{k^{l / 3}[1+P z]}[1+P z] A_{1}\left(1+P z^{-}\right) d z^{-},
$$

where $P=\left(\rho_{c}-\rho_{a}\right) / \rho_{a} \Delta z$ and where $A_{i}(x)$ is an Airy function.

The Region $b$ solution ( $C 2$ ) to the time-independent wave equation (C1) must be matched on to the Region and Region c solutions which, using the definition of $\zeta$, are

$$
f_{a}(\zeta)=I \exp \left\{1 K^{1 / 2}(\zeta-1)\right\}+R \exp \left\{-1 K^{1 / 2}(\zeta-1)\right\}
$$

and

$$
f_{c}(\zeta)=T \exp \left\{1 \frac{v_{A_{a}}}{v_{A_{c}}} K^{1 / 2}(\zeta-1)\right\}
$$

where $I, R$, and $T$ are the magnetic-induction amplitudes of the incident, reflected, and transmitted waves. Matching $f_{b}$ and $\partial f_{b} / \partial z$ to $f_{a}$ and $\partial f_{a} / \partial \zeta$ at $z=z_{0}(\zeta=1)$ yields two relations for $a_{0}$ and $a_{1}$ in terms of the incidence and reflection coefficlents $I$ and $R$ :

$$
a_{0}=\frac{S_{42}^{*}(1) I+S_{42}(1) R}{s_{1}(1) s_{4}(1)-s_{2}(1) s_{3}(1)}
$$

and

$$
a_{1}=-\frac{s_{31}^{*}(1) I+s_{31}(1) R}{s_{1}(1) s_{4}(1)-s_{2}(1) s_{3}(1)}
$$


where * denotes complex conjugate, where $s_{p q}(\zeta)=s_{p}(\zeta)+1 k^{1 / 2} s_{q}(\zeta)$, and where

$$
\begin{aligned}
& \left.S_{3}(\zeta)=\frac{\partial S_{1}}{\partial \zeta}=\sum_{n=1}^{\infty} \frac{(-K)^{n}}{(3 n-1) !} \prod_{m=1}^{n}(3 m-1)\right] \zeta^{3 n-1} \\
& S_{4}(\zeta)=\frac{\partial S_{2}}{\partial \zeta}=2 \zeta+2 \sum_{n=1}^{\infty} \frac{(-K)^{n}}{(3 n+1) !}\left[\prod_{m=1}^{n}(3 m+1)\right] \zeta^{3 n+1} .
\end{aligned}
$$

Matching $f_{b}$ and $\partial f_{b} / \partial z$ to $f_{c}$ and $\partial f_{c} / \partial \zeta$ at $z=z_{o}+\Delta z$, which is $\zeta=1+$ $\left(\rho_{c}-\rho_{a}\right) / \rho_{a}$, ylelds two relations for the reflection and transmission coefficients $R$ and $T$ in terms of the incidence coefficient $I$ :

$$
R=I \frac{1 \frac{v_{A_{a}}}{v_{A_{c}}} k^{1 / 2}\left(s_{421}^{*}-s_{312}^{*}\right)-\left(s_{423}^{*}-s_{314}^{*}\right)}{-1 \frac{v_{A_{a}}}{v_{A_{c}}} K^{1 / 2}\left(s_{421}-s_{312}\right)+\left(s_{423}-s_{314}\right)}
$$

and

$$
\begin{aligned}
& T=I \frac{s_{42}(1) s_{31}^{*}(1)-s_{42}^{*}(1) s_{31}(1)}{-1 \frac{v_{A_{a}}}{v_{A_{c}}} k^{1 / 2}\left(s_{421}-s_{312}\right)+\left(s_{423}-s_{314}\right)} \\
& \times \frac{S_{1234}\left(\zeta_{\Delta z}\right)}{S_{1234}(1)} \exp \left[-1 \frac{v_{A_{a}}}{v_{A_{C}}} K^{1 / 2}\left(\zeta_{\Delta z}-1\right)\right],
\end{aligned}
$$

where $\zeta_{\Delta z}=1+\left(\left(\rho_{c}-\rho_{a}\right) / \rho_{a}\right)$ and where $s_{p q r}=s_{p q}(1) s_{r}\left(\zeta_{\Delta z}\right)$ and $s_{1234}(\zeta)=$ $\mathrm{s}_{1}(\zeta) \mathrm{s}_{4}(\zeta)-\mathrm{s}_{2}(\zeta) \mathrm{s}_{3}(\zeta)$. These coefficients are the theoretical curves appearing In Figure 9, plotted as functions of the ramp thickness. For $\Delta z \rightarrow$ $n$, equations ( $\mathrm{C} 9 \mathrm{a}$ ) and ( $\mathrm{C} 9 \mathrm{~b})$ reduce to the Fresnel formulae for Alfven-wave magnetic-induction reflection and transmission coefficlents for discontinuities in the plasma density (see Section VII.A of Ref. 14 and Section 3.4 .5 of Ref. 6): 


$$
R=I \frac{\rho_{c}^{1 / 2}-\rho_{a}^{1 / 2}}{\rho_{c}+\rho_{a}}
$$

and

$$
T=I \frac{2 \rho_{c}^{1 / 2}}{\rho_{c}+\rho_{a}^{I / 2}}
$$

The analysis of electromagnetic-field propagation in uniformly resistive plasmas with density gradients is identical to that for collisionless plasmas. If there is uniform ohmic damping, then the undamped wave equation (38) takes the form of the telegrapher's equation (B1), with $v_{c}$ being a function of $z$ and $K$ being a constant. Taking the curl of equation (B1) and using Faraday's law (2) yie1d

$$
\frac{\partial^{2} B_{1}}{\partial t^{2}}-v_{c}^{2} \frac{\partial B_{1}}{\partial z^{2}}=\frac{\partial v_{c}^{2}}{\partial z} \frac{\partial B_{1}}{\partial z}-k \frac{\partial B_{1}}{\partial t} .
$$

With the assumption that the solution to equation (C11) is of the form $f\left(z_{w}\right) e^{-i \omega t}$, equations of the form of $(C 1)-(c 10)$ with $w$ replaced by $w(1+i(\kappa / \omega))^{1 / 2}$ result. Thus, the reflection and transmission coefficients and so forth are valid for damped field propagation when the substitution $\omega \rightarrow$ $\omega(1+1(k / \omega))^{1 / 2}$ is made. Note that the Fresnel formulae ( $\left.10 \mathrm{a}\right)$ and ( $\mathrm{ClOb}$ ) for discontinuous densities do not contain $w$ and hence will not contain $k$; the presence of uniform ohmic damping ( $k=$ constant) does not alter the reflection and transmission properties of plasma-density discontinuities to electromagnetic fields. 
The amplitude of an electromagnetic signal in a density ramp can be predicted with the approximation method of Rayleigh.15 When it is assumed that the solution to equation (40) is of the form

$$
B(z, t)=A e^{i \omega u(z)} e^{-1 \omega t}
$$

where A is a constant and $u(z)$ is a complex-valued function, equation (40) becomes the time-independent wave equation

$$
i \omega \frac{\partial^{2} u}{\partial z^{2}}-\omega^{2} \cdot\left(\frac{\partial u}{\partial z}\right)^{2}-1 \omega g(z) \frac{\partial u}{\partial z}+\frac{\omega^{2}}{v_{A}^{2}}=0,
$$

where $g(z)=(1 / \rho)(\partial \rho / \partial z)$. By expanding $u(z)$ in powers of $1 / \omega$ (see Ref. 16) via $u(z)=\sum_{n=0}^{\infty} \omega^{-n} u_{n}(z)$, and then equating matching powers of $w$ in equation (D2), the $u_{n}$ functions can be guaranteed to depend only on the medium, not on the properties of the wave. The time-independent wave equation ( 22 ) becomes

$$
\begin{aligned}
& \omega^{2}\left[-u_{0}^{2}+\frac{1}{v_{A}^{2}}\right]+w^{1}\left[1 u_{0}^{-}-2 u_{1}^{-} u_{0}^{-}-1 g u_{0}^{-}\right] \\
& +w^{0}\left[1 u_{1}^{-}-u_{1}^{-2}-2 u_{0}^{-} u_{2}^{-}-1 g u_{1}^{-}\right] \\
& +\omega^{-1}\left[1 u_{2}^{-}-2 u_{0}^{-} u_{i}^{-}-2 u_{1}^{-} u_{2}^{-}-1 g u_{2}^{-}\right]+\ldots .+=0,
\end{aligned}
$$

primes denoting differentiation with respect to $z$. The $\omega^{2}$ terms, the $\omega^{1}$ terms, and the $\omega^{0}$ terms yield, respectively,

$$
\begin{aligned}
& u_{0}(z)=u_{0}(0) \pm \int_{0}^{z} \frac{1}{v_{A}(z)} d z, \\
& u_{1}(z)=u_{1}(0)+\frac{1}{4} \log \left(\frac{\rho(0)}{\rho}\right),
\end{aligned}
$$


and

$$
u_{2}(z)=u_{2}(0) \pm \int_{0}^{z} v_{A}\left[-\frac{7}{32} g^{2}+\frac{1}{8} \frac{1}{\rho} \frac{\partial^{2} \rho}{\partial z^{2}}\right] d z .
$$

When, at the foot of the density ramp, the density-ramp solution is matched onto a uniform-medium solution $B(z=0, t)=A e^{-i \omega t}$, it is found that $u_{1}(0)=$ $u_{2}(0)=u_{3}(0)=\ldots=0$. For a linear density ramp $\rho=$ $\rho_{a}+\left[\left(\rho_{c}-\rho_{a}\right) z / \rho_{a} \Delta z\right]$, the Integrals in relations (D4) can be performed, and to order $1 / \omega^{2}$ in the expansion, the single-frequency solution within the density ramp becomes

$$
u(z, t)=A_{0}\left(\frac{\rho}{\rho(0)}\right)^{1 / 4} \exp \left[i \omega \frac{2}{3} \frac{1}{v_{A} g}+1 \frac{1}{\omega} \frac{7}{48} v_{A} g-1 \omega t\right],
$$

where the constant $A$ and exponentials of the constant of integration $u_{0}(0)$ have been combined into $A_{0}$. For the expansion technique to be valid, the magnitude of successive terms must decrease; for the $u_{1}$ and $u_{0} \cdot t e r m s$, this decrease requires that the wavelength be small compared with $16 \pi$ times the. density scale size, which for the linear ramp is $\lambda<16 \pi \Delta z$. 
In conducting media, single-frequency wave functions satisfying the telegrapher's equation (B1) have the form $E \times e^{-1 \omega t} \exp \left[ \pm 1 \frac{\omega}{v}\left(1+1 \frac{k}{\omega}\right)^{l / 2} z\right]$. For a purely transverse electromagnetic disturbance propagating with normal incidence into a planar discontinulty between two media, conserving the tangential components of the wave flelds $\vec{E}$ and $\vec{B}$ yields the electric-field reflection and transmission coefficients (see Section 6-2 of Ref. 17)

$$
\begin{aligned}
R=I \frac{v_{c 2}\left(1+1 \frac{k_{1}}{\omega}\right)^{1 / 2}-v_{c 1}\left(1+1 \frac{k_{2}}{\omega}\right)^{1 / 2}}{v_{c 2}\left(1+1 \frac{k_{1}}{\omega}\right)^{1 / 2}+v_{c 1}\left(1+1 \frac{k_{2}}{\omega}\right)^{1 / 2}} \\
T=I \frac{2 v_{c 2}\left(1+1 \frac{k_{1}}{\omega}\right)^{1 / 2}}{v_{c 2}\left(1+1 \frac{k_{1}}{\omega}\right)^{1 / 2}+v_{c 1}\left(1+1 \frac{k_{2}}{\omega}\right)^{1 / 2}}
\end{aligned}
$$

The media of incidence are characterized by the constants $v_{c l}$ and $k_{1}$ and the media of transmittance by the constants $v_{c 2}$ and $\kappa_{2}$.

These discontinuous-media coefficients are equivalent to coefficients obtained by impedance-matching arguments. At an interface at $z=0$ between two uniform media, the surface impedance $z_{8}(\omega)$ of a conductor to an electromagnetic wave with frequency $\omega$ is defined according to the relation (see Section 8.1 of Ref. 1n) $\int_{0}^{\infty} J(\omega, z) d z=E(z=0, \omega) / Z_{s}(\omega)$. Since the electric-field propagation is described by the telegrapher's equation, the relation between $\vec{J}(z, t)$ (including the displacement current) and $\vec{E}(z, t)$ is $\vec{J}=$ $(\kappa / 4 \pi)\left(c^{2} / v_{c}^{2}\right)+\vec{E}+(1 / 4 \pi)\left[\left(c^{2} / v_{c}^{2}\right)-1\right](\partial \vec{E} / \partial t)+(1 / 4 \pi)(\partial \vec{E} / \partial t)$. The electric field within the medium $(z>0)$ is of the form $\vec{E}(z, t)=$ $E(z=0, \omega) e^{-I \omega t} e^{1 k z}$, where the real and imaginary parts of $k$ are given by equations (B6a) and (B6b). W1th this electric-field form, the integration over $J$ can be performed, ylelding $z_{s}=\left(4 \pi v_{c}^{2} / c^{2}\right)\left(k_{1 m a g}-1 k_{r e a 1}\right) /(k-1 \omega)$. Since $\left(k_{\text {imag }}-1 k_{\text {real }}\right)=-1\left(k_{\text {real }}+1 k_{\text {imag }}\right)$ and since relation (B2) gives $k^{2}=$ $\left(\omega^{2} / v_{c}^{2}\right)[1+1(k / \omega)]$, the Impedance can be written

$$
z_{s}=\frac{4 \pi v_{c}}{c^{2}}\left(1+1 \frac{k}{\omega}\right)^{-1 / 2} .
$$


For $k \gg \omega, z_{s}$ becomes $z_{s} \simeq\left(4 \pi v_{c} / c^{2}\right)\left(\omega^{1 / 2} / k^{1 / 2}\right)(1-1) / \sqrt{2}$, and for $k+0$, $\mathrm{z}_{s}$ becomes $\mathrm{z}_{s}=4 \pi \mathrm{v}_{c} / \mathrm{c}^{2}$, which becomes $4 \pi / c$ in the 1 imit $\mathrm{v}_{c}+c$. This final value $(=377 \Omega$ ) is the Impedance of a vacuum to electromagnetic waves (see Section 9.5 of Ref. 18). In analogy to telegraphic circuits,18,19 the reflection and transmission coefficients for the passage of a harmonic electromagnetic wave through a planar discontinuity between two homogeneous media are conveniently written in terms of the surface impedences $Z_{s}$ of the media to the wave; with the use of Equation (E2), the electric-field reflection and transmission coefficients (Ela) and (EIb) for a discontinulty can be written

$$
R=I \frac{Z_{s_{2}}-z_{s_{1}}}{Z_{s_{2}}+z_{s_{1}}}
$$

and

$$
T=I \frac{2 z_{s_{2}}}{Z_{s_{2}}+\mathrm{Z}_{s_{1}}}
$$


1. E. Åström, "On Waves in an Ionized Gas," Ark. Fys. 2, 443 (1950).

2. J. E. Borovsky, "Numerics for the Simulation of Electromagnetic-Field propagation in Nonionized and Plasma Media," Los Alamos National Labora tory report LA-10627-MS (1986).

3. H. Alfvén, "On the Existence of Electromagnetic-Hydrodynamic Waves," Ark. Ma t., Astron. Fys. 29-2, 1 (1942).

4. H. Alfvén, "Existence of Electromagnetic-Hydrodynamic Weves," Nature 150, $405(1942)$.

5. J. H. Piddington, "The Motion of Ionized Gas in Combined Magnetic Electric and Mechanical Fields of Force", Mon. Not. R. Astron. Soc. 114,651 (1954).

6. H. Alfvén and C.-G. Falthammar, Cosmical Electrodynamics, Second Edition (Oxford, Londor, 1963).

7. D. R. Nicholson, Introduction to Plasma Theory (W1ley, New York, 1983).

8. J. C. Maxwe1l, "A Dynamical Theory of the Electromagnetic Field," Ph11. Trans. 459 (1865).

9. J. C. Maxwell, A Treatise on Electricity and Magnetism, 3rd edition (Oxford, London, $1 \overline{8} 8 \overline{2}$ ).

10. J. D. Jackson, Classica 1 Electrodynamics, 2nd edition (Wiley, New York, 1975).

11. J. S. Townsend, The Theory of Ionization of Gases by Collision, Section 11 (Constable, London, 1910).

12. J. S. Townsend, Electricity in Gases, Section 77 (Oxford, London, 1915).

13. M. Abramowitz and I. Stegun, Handbook of Ma thematica 1 Functions (Nationa 1 Bureau of Standards, Washington, $\overline{D C}, 1 \overline{964} \overline{\text {. }}$

14. S. Lundquist, "Studies in Magreto-Hydrodynamics," Ark. Fys. 프 15, 297 (1952).

15. Lord Rayleigh, "On the Propagation of Waves through a Stratifled Medium, with Special Reference to the Ouestion of Reflection," Proc. $R$. Soc. London. A86, 207 (1912).

16. H. Jeffreys, "On Certain Approximate Solutions of Linear Differential Equations of the Second Order," Proc. London Math. Soc. 23, 428 (1923).

17. I. Tolstoy, Wave Propagation (McGraw-H111, New York, 1973).

18. A. Hirose and K. E. Lonngren, Introduction to Wave Phenomena (Wiley, New York, 1985). 
19. S. Ramo, R. Whinnery, and T. van Duzer, Flelds and Waves in Communication Electronics, Section 1.16 (Wiley, New York, 1965). 